\title{
To Explore the Network Pharmacology and Molecular Docking Mechanism of Chaihu Shugan Powder with the "Same Treatment for Different Diseases" for Insomnia and Depression Based on the COVID-19 Pandemic
}

\author{
Liang Wang \\ Chinese PLA General Hospital \\ Peng Wang \\ Chinese PLA General Hospital \\ Yingfan Chen \\ Chinese PLA General Hospital \\ Chen Li \\ Chinese PLA General Hospital \\ Xuelin Wang \\ Chinese PLA General Hospital \\ Mingwei Li \\ Chinese PLA General Hospital \\ Zhenxian Luan \\ Chinese PLA General Hospital \\ Yin Zhang \\ Chinese PLA General Hospital \\ Shaodan Li ( $\square$ Isd301@126.com) \\ Chinese PLA General Hospital \\ Minghui Yang \\ Chinese PLA General Hospital
}

\section{Research Article}

Keywords: OVID-19 Pandemic, CHSGP, Network Pharmacology, Molecular Docking, Same Treatment for Different Diseases, Insomnia, Depression, Theoretical Basis

Posted Date: March 9th, 2022

DOI: https://doi.org/10.21203/rs.3.rs-1332355/v2

License: (c) (7) This work is licensed under a Creative Commons Attribution 4.0 International License. Read Full License 


\section{Abstract}

Background: The classic prescription Chaihu Shugan Powder (CHSGP) has been widely used in clinical Chinese medicine treatment and has clear clinical effects in the treatment of emotional diseases. Based on the increasing incidence of emotional diseases such as insomnia and depression in the population during the COVID-19 pandemic, we will explore the mechanism of CHSGP in the treatment of insomnia and depression with "Same Treatment for Different Diseases".

Methods: Using a bioinformatics and network pharmacology platform, protein database and STRING database, we collected CHSGP chemical composition and related target data and constructed a "component-target" action network through Gene Ontology and Kyoto Encyclopedia of Genes and Genome pathway enrichment analysis. Molecular docking technology was used to verify key active ingredients and core targets.

Results: A total of 119 active compounds of CHSGP were screened, such as quercetin, kaempferol, and $\beta$-sitosterol, and 113 common related targets overlapped with insomnia and depression. GO enrichment and KEGG pathway analysis mainly involved immune, inflammation, cell proliferation, apoptosis, endocrine and other related targets and signaling pathways. Molecular docking showed that small molecular compounds (kaempferol, luteolin, quercetin, 7-methoxy-2-methyl isoflavone and beta-sitosterol) had good binding effects with five target proteins (AKT1, IL1B, IL-6, FOS, GSK3B) to play a role in regulating immunity, the inflammatory response, cell proliferation, apoptosis, and endocrine signaling.

Conclusions: Under the context of the COVID-19 pandemic, it revealed the complex mechanism of multicomponent, multitarget, and multipathway of the classic CHSGP for insomnia and depression, laying a theoretical foundation for its clinical application of its "same treatment for different diseases".

\section{Highlights}

- Network pharmacology and molecular docking were used to explore the mechanism of CHSGP for insomnia and depression with "Same Treatment for Different Diseases".

- Among the core targets of CHSGP against AKT1, IL1B, IL-6, FOS, and GSK3B in insomnia and depression.

- The AGE-RAGE pathway, neuroactive ligand-receptor interaction, and IL-17 signaling pathway were significantly enriched based on KEGG pathway enrichment analysis.

- The compounds kaempferol, luteolin and quercetin matched well with the target proteins AKT1, IL1B, IL-6, FOS and GSK3B.

\section{Introduction}

During the global pandemic of COVID-19, economic data in various countries fell, and unemployment rates rose. During the epidemic, many people need to be isolated at home and face outstanding problems, such as work restrictions, academic delays and financial burdens [1]. Many surveys [25] have shown that medical staff are at the front line of the fight against the epidemic and under high-intensity work pressure, and they are more likely to suffer from diseases such as depression and sleep disorders. Insomnia, as a common subjective sleep disorder, is mainly manifested as having difficulty falling asleep, sleep maintenance disorder, continuous early awakening, decreased sleep quality, and total sleep time reduction, accompanied by daytime dysfunction [6]. Meanwhile, depression, as a widespread psychological disorder, is characterized by significant and lasting depression, reduced mobility, delayed thinking and cognitive functions with boring, helpless, incompetent, powerless, hopeless and worthless feelings, affecting up to $20 \%$ of the global population [7]. Studies have found that the relationship between sleep and depression is two-way, complex and obvious [8]. With the continuous development of the social economy, the prevalence and comorbidities of insomnia and depression are becoming increasingly common [9], and they often occur at the same time [10]. In particular, depression and insomnia caused by the impact of the COVID-19 epidemic have become increasingly prominent, and timely treatment cannot be waited for them [11]. Due to various adverse reactions in the clinical use of Western medicine, many studies have shown that the use of complementary and alternative medicine (CAM) in mental illnesses such as insomnia and depression is a common phenomenon [12]. In addition, compared with pure Western medicine treatment, TCM alone or in combination with Western medicine has the characteristics of quick onset, high cure rate, and fewer side effects [13].

For thousands of years, TCM and its compounds have been used to treat mental illnesses such as insomnia and depression [14]. The earliest record of CHSGP was found in "Jingyue Quanshu" and described it as a classic TCM prescription developed from Sini Soup. Its main components include Chaihu, Baishao, Chuanxiong, Chenpi, Xiangfu, Zhike, and Gancao. The principal active ingredients of CHSGP are monoterpene glycosides, glucose gallic acid, phenolic compounds, lactones, flavonoids and triterpene saponins [15]. CHSGP has the effects of soothing the liver, promoting qi, activating blood and relieving pain and is often used to treat depression, insomnia, anxiety, chronic gastritwas, hepatitis and other diseases [16]. As the name suggests, "Same Treatment for Different Diseases" means different diseases can be treated the same. The theory of TCM classifies insomnia and depression into the categories of "insomnia" and "stagnation syndrome", respectively. The classification often involves multiple organs, such as the liver, spleen, lung, and heart, and can often be treated for liver depression and qi stagnation. This is the fundamental principle of treatment in the "Same Treatment for Different Diseases" of CHSGP. CHSGP matches the menstrual flow of insomnia and depression and can better exert its therapeutic effect. Pharmacological studies have found that the antidepressant pharmacological properties of CHSGP also have a certain 
therapeutic effect on insomnia and mainly regulate the neuroendocrine immune network, exerting anti-inflammatory and antioxidative stress effects [17].

Due to its multicomponent and multitarget characteristics, TCM makes in-depth research difficult. Network pharmacology explores the material basis of TCM treatment of diseases from a systemic and holwastic perspective by constructing a "drug-target-gene-disease" network and molecular mechanism [18]. This finding is consistent with the overall concept of TCM and Same Treatment for Different Diseases. It provides new ideas, new methods and new ways for TCM mining and analysis and shows the visualization and relevance of data. Its fast and accurate data mining classification and positioning method has been widely recognized $[19,20]$.

Therefore, this study constructed a "component-target-pathway-disease" association network of CHSGP for insomnia and depression with "Same Treatment for Different Diseases" through network pharmacology and molecular docking. The mechanism of action of multiple targets and pathways provides a certain theoretical basis for clinical applications in treating insomnia-depression during the COVID-19 pandemic.

\section{Materials And Methods}

\subsection{Research ideas}

Under the context of the COVID-19 pandemic, this study was based on network pharmacology and molecular docking to explore the research ideas in the mechanism of CHSGP "treating different diseases with the same treatment" insomnia and depression. The experimental flow is shown in Fig. 1.

\subsection{Screening the effective ingredients and targets of CHSGP}

Through the TCMSP database [21] (https://tcmspw.com/tcmsp.php), the pharmacological analysis platform of the Chinese medicine system, OB $\geq$ $30 \%, \mathrm{DL} \geq 0.18$, was used as the ingredient screening criteria to search for Chaihu, Baishao, Chuanxiong, Chenpi, Xiangfu, Zhike, and Gancao. The components of the included compounds were predicted and extracted through the TCMSP database. All targets were corrected by the UniProt database [22](https://www.uniprot.org/), and nonhuman targets were removed to obtain "official genes symbol" of all targets. According to the theory of TCM, the effective active ingredient targets of TCM have the characteristics of selective distribution in the body, which is basically consistent with the visceral relationship of the corresponding meridian homing. A Venn diagram was used to show the intersection of the meridian targets of TCM.

\subsection{Construction and analysis of the protein interaction network (PPI)}

We inputed the common targets of drugs and diseases into the String database [23] (https://string-db.org/cgi/input.pl), used the multiple proteins function of the database to construct a PPI network, and set the biological species as "Homo sapiens" at the same time. Finally, a combined score > 0.4 was used as the screening standard to obtain the PPI network model of the intersection target.

\subsection{Topological analysis and cluster analysis}

In this study, the screened PPI network information was imported into Cytoscape 3.8.0 for network topology analysis, and common key targets were screened based on the degree value and betweenness centrality. We imported the constructed PPI network into Cytoscape 3.8.0, analyzed gene clusters and screened core genes through MCODE module analysis [24].

\subsection{Heatmaps of key target genes expressed in organs and tissues}

We inputed the key target genes selected by topological analysis into the BioGPS database (http://biogps.org/\#goto=welcome) and set "current layout" as the "default layout", downloaded the organ and tissue expression data corresponding to the first 20 key target genes. We standardized and visualized the data and plotted the expression heatmaps of the top 20 key target genes in major organs and tissues.

\subsection{Visualization of the component-disease-target network and screening of key TCM components}

To better understand the complex interactions between components, diseases and corresponding targets, we built a component-disease-target network diagram based on the included components, disease treatments, and targets and introduced Cytoscape 3.8 .0 and drew a visual network diagram. We imported the component-disease-target network diagram into Cytoscape 3.8.0 for topological analysis and sorted the components by degree. The higher the degree value, the more important the component (we selected the component greater than the average degree value as the key component for subsequent follow-up research).

\subsection{GO enrichment analysis and KEGG pathways analysis}

The common targets of drugs and diseases were analyzed for GO enrichment and KEGG pathway analysis. GO enrichment included biological process (BP), molecular function (MF), and cell component (CC) enrichment. Quoting the String database and filtering the items with the corrected $P$ 
value $<0.05$. At the same time, R 4.0.3 software was used to install and reference clusterProfiler, enrichplot, and ggplot2 packages and draw histograms and bubble charts. We used the Sangerbox tool to visually analyze the drug-disease KEGG signaling pathway and draw a chord diagram.

\subsection{Composition-disease-Pathway-Target Network Construction}

We imported the component-disease-pathway-target network file into Cytoscape 3.8.0 to draw the path network diagram, which more intuitively showed the characteristics of the multicomponent-multitarget effect of the TCM active ingredients in the treatment of diseases.

\subsection{Molecular docking verification}

We imported the constructed component-disease-target network diagram to screen the key component compounds, such as kaempferol, luteolin, quercetin, beta-sitosterol, and 7-methoxy-2-methyl isoflavone, into the PubChem database (https://pubchem.ncbi.nlm.nih.gov/), queried the MOL2 format, and imported Schrodinger software to establish a database, which was prepared as a ligand molecular database for molecular docking [25] We downloaded the target protein crystal structure from the protein database (https://www.rcsb.org/), processed it with the Maestro11.9 platform, processed the protein with Schrodinger's Protein Preparation Wizard [26], and prepared the protein receptor [27]. All compounds were prepared according to the default settings of the LigPre module [28]. Finally, molecular docking and screening were carried out by the SP method, and Pymol2.1 was used for visual analysis.

\section{Results}

\subsection{Inquiry and analysis of TCM ingredients and target information}

Using the TCMSP database and setting $\mathrm{OB} \geq 30 \%$ and $\mathrm{DL} \geq 0.18$ as the screening conditions, the components of the included compounds were predicted by the TCMSP database. All targets were calibrated by the UniProt database, and nonhuman targets were removed. After summarizing and deleting the duplicates, we got 17 compound components and 179 targets of Chaihu, 13 compound components and 86 targets of Baishao, 5 compound components and 65 targets of Chenpi,7 compound components and 30 targets of Chuanxiong, Gancao has 93 compound components and 216 targets, Xiangfu has 18 compound components and 210 targets, Zhike has 5 compound components and 88 targets. The analysis of Guijing in CHSGP showed that Chaihu, Baishao, Chuanxiong, and Xiangfu belonged to the liver channel with 18 common targets; Baishao, Xiangfu, Zhike, Chenpi, and Gancao belonged to the spleen channel with 27 common targets; Chaihu, Chenpi, and Gancao belonged to the lung channel with 39 common targets, as shown in Table 1. The above data showed that each Chinese medicine of CHSGS mainly belonged to the liver, spleen, and lung meridians, which were also in line with the location and pathogenesis of insomnia and depression and reflected the connotation of "Same Treatment for Different Diseases", as shown in Fig. 2. 
Table 1

Basic information of active ingredients of CHSGS

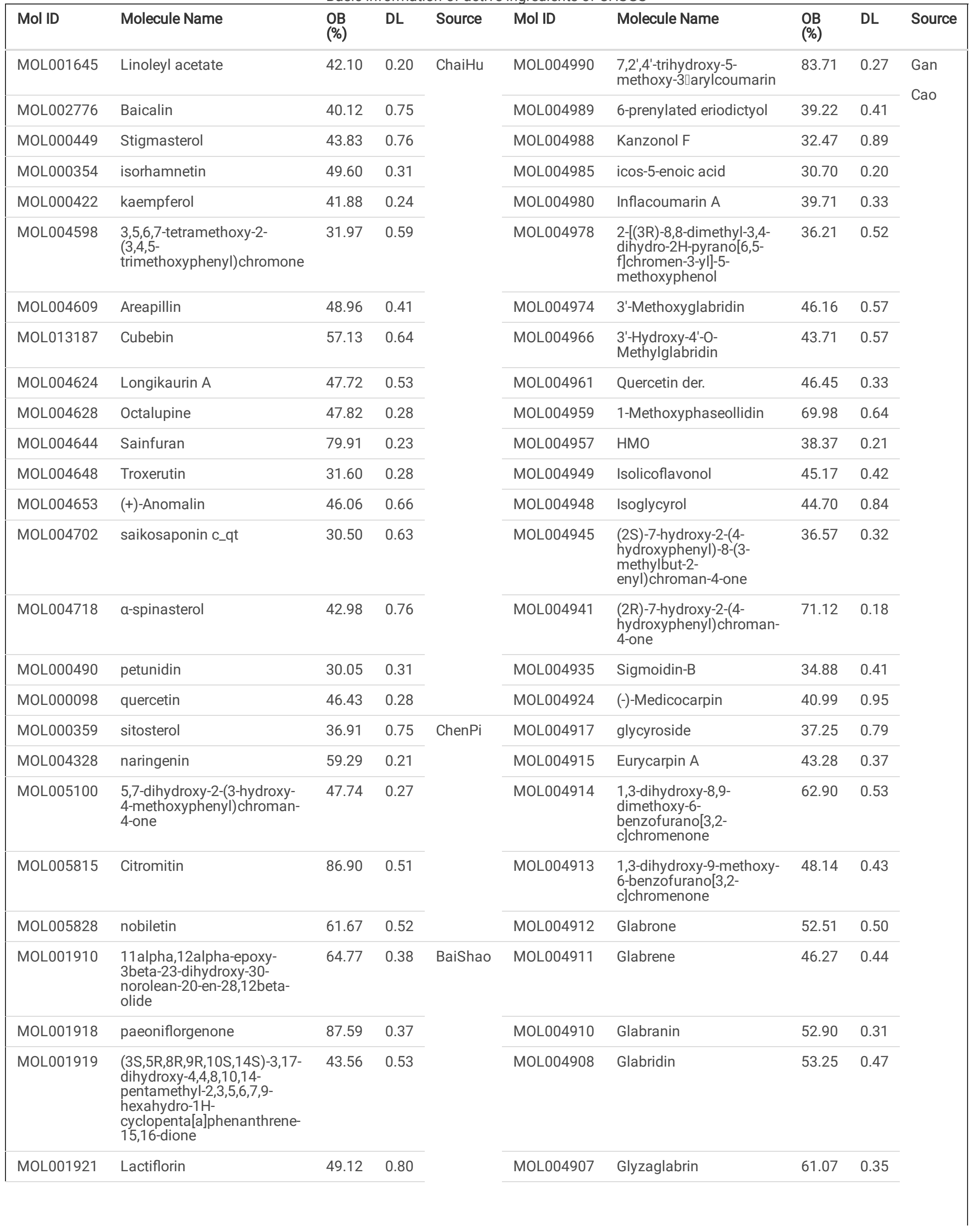




\begin{tabular}{|c|c|c|c|c|c|c|c|c|c|}
\hline Mol ID & Molecule Name & $\begin{array}{l}\mathrm{OB} \\
(\%)\end{array}$ & DL & Source & Mol ID & Molecule Name & $\begin{array}{l}\text { OB } \\
(\%)\end{array}$ & DL & Source \\
\hline MOL001924 & paeoniflorin & 53.87 & 0.79 & & MOL004905 & $\begin{array}{l}\text { 3,22-Dihydroxy-11-oxo- } \\
\text { delta(12)-oleanene-27- } \\
\text { alpha-methoxycarbonyl- } \\
\text { 29-oic acid }\end{array}$ & 34.32 & 0.55 & \\
\hline MOL001925 & paeoniflorin_qt & 68.18 & 0.40 & & MOL004904 & licopyranocoumarin & 80.36 & 0.65 & \\
\hline MOL001928 & albiflorin_qt & 66.64 & 0.33 & & MOL004903 & liquiritin & 65.69 & 0.74 & \\
\hline MOL001930 & benzoyl paeoniflorin & 31.27 & 0.75 & & MOL004898 & $\begin{array}{l}\text { (E)-3-[3,4-dihydroxy-5-(3- } \\
\text { methylbut-2- } \\
\text { enyl)phenyl]-1-(2,4- } \\
\text { dihydroxyphenyl)prop-2- } \\
\text { en-1-one }\end{array}$ & 46.27 & 0.31 & \\
\hline MOL000211 & Mairin & 55.38 & 0.78 & & MOL004891 & shinpterocarpin & 80.30 & 0.73 & \\
\hline MOL000358 & beta-sitosterol & 36.91 & 0.75 & & MOL004885 & licoisoflavanone & 52.47 & 0.54 & \\
\hline MOL000359 & sitosterol & 36.91 & 0.75 & & MOL004884 & Licoisoflavone B & 38.93 & 0.55 & \\
\hline MOL000422 & kaempferol & 41.88 & 0.24 & & MOL004883 & Licoisoflavone & 41.61 & 0.42 & \\
\hline MOL000492 & $(+)$-catechin & 54.83 & 0.24 & & MOL004882 & Licocoumarone & 33.21 & 0.36 & \\
\hline MOL001494 & Mandenol & 42.00 & 0.19 & Chuan & MOL004879 & Glycyrin & 52.61 & 0.47 & \\
\hline MOL002135 & Myricanone & 40.60 & 0.51 & Xiong & MOL004866 & $\begin{array}{l}\text { 2-(3,4- } \\
\text { dihydroxyphenyl)-5,7- } \\
\text { dihydroxy-6-(3- } \\
\text { methylbut-2- } \\
\text { enyl)chromone }\end{array}$ & 44.15 & 0.41 & \\
\hline MOL002140 & Perlolyrine & 65.95 & 0.27 & & MOL004864 & $\begin{array}{l}\text { 5,7-dihydroxy-3-(4- } \\
\text { methoxyphenyl)-8-(3- } \\
\text { methylbut-2- } \\
\text { enyl)chromone }\end{array}$ & 30.49 & 0.41 & \\
\hline MOL002151 & senkyunone & 47.66 & 0.24 & & MOL004863 & $\begin{array}{l}\text { 3-(3,4- } \\
\text { dihydroxyphenyl)-5,7- } \\
\text { dihydroxy-8-(3-- } \\
\text { methylbut-2-- } \\
\text { enyl)chromone }\end{array}$ & 66.37 & 0.41 & \\
\hline MOL002157 & wallichilide & 42.31 & 0.71 & & MOL004860 & licorice glycoside $\mathrm{E}$ & 32.89 & 0.27 & \\
\hline MOL000359 & sitosterol & 36.91 & 0.75 & & MOL004857 & Gancaonin B & 48.79 & 0.45 & \\
\hline MOL000433 & FA & 68.96 & 0.71 & & MOL004856 & Gancaonin A & 51.08 & 0.4 & \\
\hline MOL003044 & Chryseriol & 35.85 & 0.27 & Xiang & MOL004855 & Licoricone & 63.58 & 0.47 & \\
\hline MOL000354 & isorhamnetin & 49.60 & 0.31 & $\mathrm{Fu}$ & MOL004849 & $\begin{array}{l}\text { 3-(2,4- } \\
\text { dihydroxyphenyl)-8-(1,1- } \\
\text { dimethylprop-2-enyl)-7- } \\
\text { hydroxy-5-methoxy- } \\
\text { coumarin }\end{array}$ & 59.62 & 0.43 & \\
\hline MOL003542 & 8-Isopentenyl-kaempferol & 38.04 & 0.39 & & MOL004848 & licochalcone G & 49.25 & 0.32 & \\
\hline MOL000358 & beta-sitosterol & 36.91 & 0.75 & & MOL004841 & Licochalcone B & 76.76 & 0.19 & \\
\hline MOL000359 & sitosterol & 36.91 & 0.75 & & MOL004838 & $\begin{array}{l}\text { 8-(6-hydroxy-2- } \\
\text { benzofuranyl)-2,2- } \\
\text { dimethyl-5-chromenol }\end{array}$ & 58.44 & 0.38 & \\
\hline MOL004027 & $\begin{array}{l}\text { 1,4-Epoxy-16- } \\
\text { hydroxyheneicos- } \\
1,3,12,14,18 \text {-pentaene }\end{array}$ & 45.10 & 0.24 & & MOL004835 & Glypallichalcone & 61.60 & 0.19 & \\
\hline MOL004053 & Isodalbergin & 35.45 & 0.20 & & MOL004833 & Phaseolinisoflavan & 32.01 & 0.45 & \\
\hline MOL004058 & Khell & 33.19 & 0.19 & & MOL004829 & Glepidotin B & 64.46 & 0.34 & \\
\hline MOL004059 & khellol glucoside & 74.96 & 0.72 & & MOL004828 & Glepidotin A & 44.72 & 0.35 & \\
\hline MOL010489 & Resivit & 30.84 & 0.27 & & MOL004827 & Semilicoisoflavone B & 48.78 & 0.55 & \\
\hline
\end{tabular}




\begin{tabular}{|c|c|c|c|c|c|c|c|c|c|}
\hline Mol ID & Molecule Name & $\begin{array}{l}\text { OB } \\
(\%)\end{array}$ & DL & Source & Mol ID & Molecule Name & $\begin{array}{l}\mathrm{OB} \\
(\%)\end{array}$ & DL & Source \\
\hline MOL004068 & rosenonolactone & 79.84 & 0.37 & & MOL004824 & $\begin{array}{l}\text { (2S)-6-(2,4- } \\
\text { dihydroxyphenyl)-2-(2- } \\
\text { hydroxypropan-2-yl)-4- } \\
\text { methoxy-2,3- } \\
\text { dihydrofuro[3,2- } \\
\text { g]chromen-7-one }\end{array}$ & 60.25 & 0.63 & \\
\hline MOL004071 & Hyndarin & 73.94 & 0.64 & & MOL004820 & kanzonols W & 50.48 & 0.52 & \\
\hline MOL004074 & stigmasterol glucoside_qt & 43.83 & 0.76 & & MOL004815 & $\begin{array}{l}\text { (E)-1-(2,4- } \\
\text { dihydroxyphenyl)-3-(2,2- } \\
\text { dimethylchromen-6- } \\
\text { yl)prop-2-en-1-one }\end{array}$ & 39.62 & 0.35 & \\
\hline MOL004077 & sugeonyl acetate & 45.08 & 0.20 & & MOL004814 & Isotrifoliol & 31.94 & 0.42 & \\
\hline MOL000422 & kaempferol & 41.88 & 0.24 & & MOL004811 & Glyasperin C & 45.56 & 0.4 & \\
\hline MOL000449 & Stigmasterol & 43.83 & 0.76 & & MOL004810 & glyasperin F & 75.84 & 0.54 & \\
\hline MOL000006 & luteolin & 36.16 & 0.25 & & MOL004808 & glyasperin B & 65.22 & 0.44 & \\
\hline MOL000098 & quercetin & 46.43 & 0.28 & & MOL004806 & euchrenone & 30.29 & 0.57 & \\
\hline MOL013381 & Marmin & 38.23 & 0.31 & ZhiKe & MOL004805 & $\begin{array}{l}\text { (2S)-2-[4-hydroxy-3-(3- } \\
\text { methylbut-2- } \\
\text { enyl)phenyl]-8,8- } \\
\text { dimethyl-2,3- } \\
\text { dihydropyrano[2,3- } \\
\text { f]chromen-4-one }\end{array}$ & 31.79 & 0.72 & \\
\hline MOL002341 & Hesperetin & 70.31 & 0.27 & & MOL004328 & naringenin & 59.29 & 0.21 & \\
\hline MOL000358 & beta-sitosterol & 36.91 & 0.75 & & MOL003896 & $\begin{array}{l}\text { 7-Methoxy-2-methyl } \\
\text { isoflavone }\end{array}$ & 42.56 & 0.2 & \\
\hline MOL004328 & naringenin & 59.29 & 0.21 & & MOL003656 & Lupiwighteone & 51.64 & 0.37 & \\
\hline MOL005828 & nobiletin & 61.67 & 0.52 & & MOL002844 & Pinocembrin & 64.72 & 0.18 & \\
\hline MOL005020 & dehydroglyasperins $\mathrm{C}$ & 53.82 & 0.37 & Gan & MOL002565 & Medicarpin & 49.22 & 0.34 & \\
\hline MOL005018 & Xambioona & 54.85 & 0.87 & Cao & MOL002311 & Glycyrol & 90.78 & 0.67 & \\
\hline MOL005017 & Phaseol & 78.77 & 0.58 & & MOL001792 & DFV & 32.76 & 0.18 & \\
\hline MOL005016 & Odoratin & 49.95 & 0.3 & & MOL001484 & Inermine & 75.18 & 0.54 & \\
\hline MOL005013 & $\begin{array}{l}\text { 18a-hydroxyglycyrrhetic } \\
\text { acid }\end{array}$ & 41.16 & 0.71 & & MOL000500 & Vestitol & 74.66 & 0.21 & \\
\hline MOL005012 & Licoagroisoflavone & 57.28 & 0.49 & & MOL000497 & licochalcone a & 40.79 & 0.29 & \\
\hline MOL005008 & Glycyrrhiza flavonol A & 41.28 & 0.6 & & MOL000422 & kaempferol & 41.88 & 0.24 & \\
\hline MOL005007 & Glyasperins M & 72.67 & 0.59 & & MOL000417 & Calycosin & 47.75 & 0.24 & \\
\hline MOL005003 & Licoagrocarpin & 58.81 & 0.58 & & MOL000392 & formononetin & 69.67 & 0.21 & \\
\hline MOL005001 & Gancaonin H & 50.10 & 0.78 & & MOL000359 & sitosterol & 36.91 & 0.75 & \\
\hline MOL005000 & Gancaonin G & 60.44 & 0.39 & & MOL000354 & isorhamnetin & 49.60 & 0.31 & \\
\hline MOL004996 & gadelaidic acid & 30.70 & 0.20 & & MOL000239 & Jaranol & 50.83 & 0.29 & \\
\hline MOL004993 & 8-prenylated eriodictyol & 53.79 & 0.4 & & MOL000211 & Mairin & 55.38 & 0.78 & \\
\hline MOL004991 & $\begin{array}{l}\text { 7-Acetoxy-2- } \\
\text { methylisoflavone }\end{array}$ & 38.92 & 0.26 & & MOL000098 & quercetin & 46.43 & 0.28 & \\
\hline MOL005020 & dehydroglyasperins $\mathrm{C}$ & 53.82 & 0.37 & & & & & & \\
\hline
\end{tabular}

\subsection{Disease targets search}

We searched human genes through the GeneCards, NCBI and OMIM databases, among which depression obtained 12995 related targets in the GeneCards database, 556 related targets in the NCBI database and 3 related targets in the OMIM database. Insomnia obtained 2589 related targets 
in the GeneCards database, 50 related targets in the NCBI database, and 4 related targets in the OMIM database.

\subsection{Venn diagram of common targets in drugs and diseases}

We inputed the selected drug targets and disease targets into Venn diagram production software (Venny 2.1) and obtained 113 common targets, which were used as common predictive targets for the following pathway enrichment analysis, as shown in Fig. 3.

\subsection{Construction and analysis of PPI network}

There were 113 nodes, 1365 edges, and an average degree of 24.2 in the network. Figure 4A shows the PPI network diagram exported from the STRING website, and Fig. 4B shows the PPI network diagram drawn by Cytoscape software. The color and size of the nodes in Fig. 4B were adjusted according to the degree value. The larger the color, the redder the color and the greater the degree value. Thickness from thick to thin represents the edge betweenness from large to small.

\subsection{Topological analysis and MCODE cluster analysis}

\subsubsection{Topological analysis}

We imported the PPI network into Cystoscape3.8.0 [29], used the Network Analyzer tool to perform topology analysis, and sorted by degree; the greater the degree value, the greater the role of the node in the network graph. We selected genes with scores greater than the average score as key targets. A total of 42 key targets were screened, and the top 20 targets were listed as AKT1, IL6, IL1B, CASP3, MAPK3, PPARG, MMP9, CXCL8, IL10, HSP90AA1, ESR1, FOS, HIFIA, CREB1, NOS3, HMOX1, MMP2, CCND1, ERBB2 and CASP8. We used R 4.0 .3 to draw pictures of the first top target points, where the abscissa was the degree value of each target point, as shown in Fig. 5.

\subsubsection{MCODE cluster analysis}

We imported the constructed PPI network into Cytoscape 3.8.0 and opened the MCODE module [24] to analyze gene clusters and screened core targets to obtain 7 gene clusters and 7 core genes (FOS, GABRA2, GSK3B, PON1, APOB, CYP1B1, ADRA1A). The specific results are shown in Table 2.

Table 2. MCODE cluster analysis information table for the common targets of CHSGP, insomnia and depression

5

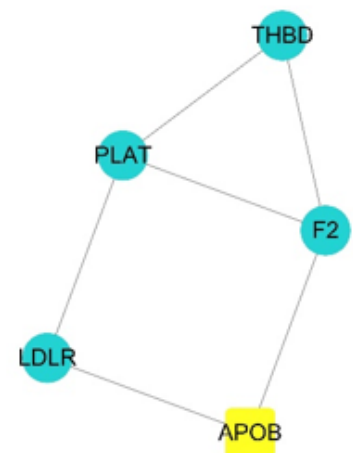

6

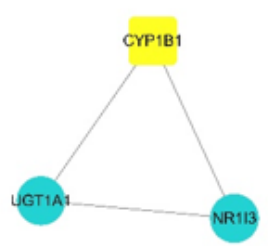

7

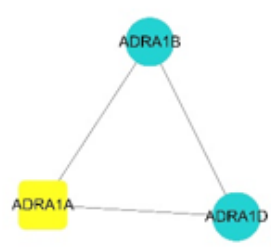

$5 \quad 6$

6 APOB, THBD, F2, PLAT, LDLR

3

3

NR1I3, UGT1A1, CYP1B1

3
ADRA1A, ADRA1D, ADRA1B

\subsection{Constructing a composition-disease target network and screening key ingredients}


To better understand the complex interactions among ingredients, diseases and corresponding targets, we constructed an "ingredient-disease-target" network based on the included ingredients, the treatment of diseases and the action targets. We screened 119 core components, such as MOL000098 (quercetin), MOL000422 (kaempferol), MOL000358 ( $\beta$-sitosterol), MOL003896 (7-methoxy-2-methyl isoflavone), and MOL000006 (luteolin), and 113 core targets, such as AKT1, IL6, IL1B, CASP3, and MAPK3. We imported the component-disease-target network into Cytoscape 3.8.0 for topological analysis. The degree was sorted according to the ingredients. The higher the degree, the more important the ingredient, and the results are shown in Table 2. Table 3 shows that the top 5 compounds with a median value of the network were MOL000098 (quercetin),

MOL000422 (kaempferol), MOL000358 ( $\beta$-sitosterol), MOL003896 (7-methoxy-2-methyl) isoflavones), and MOL000006 (luteolin), which could provide small drug compounds for subsequent molecular docking. 


\begin{tabular}{|c|c|c|c|c|c|}
\hline MOL ID & Name & $\begin{array}{l}\text { Average } \\
\text { Shortest } \\
\text { Path } \\
\text { Length }\end{array}$ & $\begin{array}{l}\text { Betweenness } \\
\text { Centrality }\end{array}$ & $\begin{array}{l}\text { Closeness } \\
\text { Centrality }\end{array}$ & Degree \\
\hline MOL000098 & quercetin & 1.958159 & 0.116804 & 0.510684 & 67 \\
\hline MOL000422 & kaempferol & 2.242678 & 0.039975 & 0.445896 & 33 \\
\hline MOL000358 & beta-sitosterol & 2.451883 & 0.020367 & 0.40785 & 26 \\
\hline MOL003896 & 7-Methoxy-2-methyl isoflavone & 2.351464 & 0.011754 & 0.425267 & 23 \\
\hline MOL000006 & luteolin & 2.460251 & 0.012164 & 0.406463 & 21 \\
\hline MOL004328 & naringenin & 2.426778 & 0.023098 & 0.412069 & 20 \\
\hline MOL000354 & isorhamnetin & 2.376569 & 0.012626 & 0.420775 & 19 \\
\hline MOL002565 & Medicarpin & 2.41841 & 0.008852 & 0.413495 & 19 \\
\hline MOL000449 & Stigmasterol & 2.711297 & 0.00895 & 0.368827 & 18 \\
\hline MOL004071 & Hyndarin & 2.543933 & 0.008647 & 0.393092 & 18 \\
\hline MOL000392 & formononetin & 2.41841 & 0.004622 & 0.413495 & 17 \\
\hline MOL000500 & Vestitol & 2.451883 & 0.004071 & 0.40785 & 15 \\
\hline MOL004959 & 1-Methoxyphaseollidin & 2.443515 & 0.002943 & 0.409247 & 15 \\
\hline MOL005828 & nobiletin & 2.460251 & 0.012514 & 0.406463 & 15 \\
\hline MOL000497 & licochalcone a & 2.460251 & 0.004353 & 0.406463 & 14 \\
\hline MOL004835 & Glypallichalcone & 2.460251 & 0.002517 & 0.406463 & 14 \\
\hline MOL004891 & shinpterocarpin & 2.460251 & 0.003491 & 0.406463 & 14 \\
\hline MOL004991 & 7-Acetoxy-2-methylisoflavone & 2.435146 & 0.003242 & 0.410653 & 14 \\
\hline MOL005003 & Licoagrocarpin & 2.443515 & 0.002204 & 0.409247 & 14 \\
\hline MOL004978 & $\begin{array}{l}\text { 2-[(3R)-8,8-dimethyl-3,4-dihydro-2H-pyrano[6,5-f]chromen-3-yl]-5- } \\
\text { methoxyphenol }\end{array}$ & 2.476987 & 0.001844 & 0.403716 & 13 \\
\hline MOL004957 & HMO & 2.485356 & 0.001897 & 0.402357 & 12 \\
\hline MOL002135 & Myricanone & 2.476987 & 0.006864 & 0.403716 & 11 \\
\hline MOL004857 & Gancaonin B & 2.493724 & 0.001127 & 0.401007 & 11 \\
\hline MOL004966 & 3'-Hydroxy-4'-0-Methylglabridin & 2.493724 & 0.001083 & 0.401007 & 11 \\
\hline MOL004974 & 3'-Methoxyglabridin & 2.493724 & 9.93E-04 & 0.401007 & 11 \\
\hline MOL005007 & Glyasperins M & 2.502092 & 0.001827 & 0.399666 & 11 \\
\hline MOL003542 & 8-Isopentenyl-kaempferol & 2.468619 & 0.003335 & 0.405085 & 10 \\
\hline MOL004808 & glyasperin B & 2.518828 & $7.86 \mathrm{E}-04$ & 0.39701 & 10 \\
\hline MOL004828 & Glepidotin A & 2.493724 & 0.001075 & 0.401007 & 10 \\
\hline MOL004833 & Phaseolinisoflavan & 2.543933 & $8.73 \mathrm{E}-04$ & 0.393092 & 10 \\
\hline MOL004908 & Glabridin & 2.543933 & 8.73E-04 & 0.393092 & 10 \\
\hline MOL000417 & Calycosin & 2.51046 & $5.90 \mathrm{E}-04$ & 0.398333 & 9 \\
\hline MOL001484 & Inermine & 2.518828 & 0.001288 & 0.39701 & 9 \\
\hline MOL002844 & Pinocembrin & 2.502092 & 0.001513 & 0.399666 & 9 \\
\hline MOL004811 & Glyasperin C & 2.527197 & 5.57E-04 & 0.395695 & 9 \\
\hline MOL004824 & $\begin{array}{l}\text { (2S)-6-(2,4-dihydroxyphenyl)-2-(2-hydroxypropan-2-yl)-4-methoxy-2,3- } \\
\text { dihydrofuro[3,2-g]chromen-7-one }\end{array}$ & 2.585774 & $5.66 \mathrm{E}-04$ & 0.386731 & 9 \\
\hline
\end{tabular}




\begin{tabular}{|c|c|c|c|c|c|}
\hline MOL ID & Name & $\begin{array}{l}\text { Average } \\
\text { Shortest } \\
\text { Path } \\
\text { Length }\end{array}$ & $\begin{array}{l}\text { Betweenness } \\
\text { Centrality }\end{array}$ & $\begin{array}{l}\text { Closeness } \\
\text { Centrality }\end{array}$ & Degree \\
\hline MOL004841 & Licochalcone B & 2.51046 & $5.90 \mathrm{E}-04$ & 0.398333 & 9 \\
\hline MOL004849 & $\begin{array}{l}\text { 3-(2,4-dihydroxyphenyl)-8-(1,1-dimethylprop-2-enyl)-7-hydroxy-5-methoxy- } \\
\text { coumarin }\end{array}$ & 2.527197 & $6.08 \mathrm{E}-04$ & 0.395695 & 9 \\
\hline MOL004856 & Gancaonin A & 2.527197 & 5.57E-04 & 0.395695 & 9 \\
\hline MOL004885 & licoisoflavanone & 2.518828 & $5.43 \mathrm{E}-04$ & 0.39701 & 9 \\
\hline MOL004907 & Glyzaglabrin & 2.518828 & $6.37 \mathrm{E}-04$ & 0.39701 & 9 \\
\hline MOL004911 & Glabrene & 2.51046 & $5.90 \mathrm{E}-04$ & 0.398333 & 9 \\
\hline MOL004912 & Glabrone & 2.51046 & $6.39 \mathrm{E}-04$ & 0.398333 & 9 \\
\hline MOL004941 & (2R)-7-hydroxy-2-(4-hydroxyphenyl)chroman-4-one & 2.502092 & 0.001513 & 0.399666 & 9 \\
\hline MOL005000 & Gancaonin G & 2.527197 & $6.58 \mathrm{E}-04$ & 0.395695 & 9 \\
\hline MOL001792 & DFV & 2.527197 & $9.10 \mathrm{E}-04$ & 0.395695 & 8 \\
\hline MOL003044 & Chryseriol & 2.527197 & 0.001336 & 0.395695 & 8 \\
\hline MOL003656 & Lupiwighteone & 2.535565 & $3.99 \mathrm{E}-04$ & 0.394389 & 8 \\
\hline MOL004053 & Isodalbergin & 2.535565 & 0.001437 & 0.394389 & 8 \\
\hline MOL004810 & glyasperin F & 2.527197 & $3.69 \mathrm{E}-04$ & 0.395695 & 8 \\
\hline MOL004815 & (E)-1-(2,4-dihydroxyphenyl)-3-(2,2-dimethylchromen-6-yl)prop-2-en-1-one & 2.535565 & 4.09E-04 & 0.394389 & 8 \\
\hline MOL004827 & Semilicoisoflavone B & 2.535565 & 4.73E-04 & 0.394389 & 8 \\
\hline MOL004848 & licochalcone G & 2.552301 & $3.64 \mathrm{E}-04$ & 0.391803 & 8 \\
\hline MOL004884 & Licoisoflavone B & 2.594142 & $3.79 \mathrm{E}-04$ & 0.385484 & 8 \\
\hline MOL004915 & Eurycarpin A & 2.535565 & 3.99E-04 & 0.394389 & 8 \\
\hline MOL004945 & (2S)-7-hydroxy-2-(4-hydroxyphenyl)-8-(3-methylbut-2-enyl)chroman-4-one & 2.527197 & $5.67 \mathrm{E}-04$ & 0.395695 & 8 \\
\hline MOL004961 & Quercetin der. & 2.527197 & $3.69 \mathrm{E}-04$ & 0.395695 & 8 \\
\hline MOL004990 & 7,2',4'-trihydroxy-5-methoxy-3ロarylcoumarin & 2.527197 & $3.69 \mathrm{E}-04$ & 0.395695 & 8 \\
\hline MOL005016 & Odoratin & 2.527197 & $3.69 \mathrm{E}-04$ & 0.395695 & 8 \\
\hline MOL002311 & Glycyrol & 2.60251 & $3.46 \mathrm{E}-04$ & 0.384244 & 7 \\
\hline MOL004814 & Isotrifoliol & 2.560669 & $3.60 \mathrm{E}-04$ & 0.390523 & 7 \\
\hline MOL004820 & kanzonols W & 2.543933 & $2.55 \mathrm{E}-04$ & 0.393092 & 7 \\
\hline MOL004863 & 3-(3,4-dihydroxyphenyl)-5,7-dihydroxy-8-(3-methylbut-2-enyl)chromone & 2.543933 & $3.29 \mathrm{E}-04$ & 0.393092 & 7 \\
\hline MOL004864 & 5,7-dihydroxy-3-(4-methoxyphenyl)-8-(3-methylbut-2-enyl)chromone & 2.560669 & $1.78 \mathrm{E}-04$ & 0.390523 & 7 \\
\hline MOL004879 & Glycyrin & 2.60251 & 3.49E-04 & 0.384244 & 7 \\
\hline MOL004883 & Licoisoflavone & 2.543933 & $4.31 \mathrm{E}-04$ & 0.393092 & 7 \\
\hline MOL004904 & licopyranocoumarin & 2.60251 & $3.93 \mathrm{E}-04$ & 0.384244 & 7 \\
\hline MOL004949 & Isolicoflavonol & 2.543933 & 3.29E-04 & 0.393092 & 7 \\
\hline MOL004980 & Inflacoumarin A & 2.51046 & $5.69 \mathrm{E}-04$ & 0.398333 & 7 \\
\hline MOL005008 & Glycyrrhiza flavonol A & 2.560669 & $2.68 \mathrm{E}-04$ & 0.390523 & 7 \\
\hline MOL005012 & Licoagroisoflavone & 2.60251 & $2.55 \mathrm{E}-04$ & 0.384244 & 7 \\
\hline MOL005017 & Phaseol & 2.543933 & $4.31 \mathrm{E}-04$ & 0.393092 & 7 \\
\hline MOL005020 & dehydroglyasperins C & 2.552301 & 3.07E-04 & 0.391803 & 7 \\
\hline
\end{tabular}

Page 11/25 


\begin{tabular}{|c|c|c|c|c|c|}
\hline MOL ID & Name & $\begin{array}{l}\text { Average } \\
\text { Shortest } \\
\text { Path } \\
\text { Length }\end{array}$ & $\begin{array}{l}\text { Betweenness } \\
\text { Centrality }\end{array}$ & $\begin{array}{l}\text { Closeness } \\
\text { Centrality }\end{array}$ & Degree \\
\hline MOL000359 & sitosterol & 2.527197 & 0.005143 & 0.395695 & 6 \\
\hline MOL000490 & petunidin & 2.543933 & 0.001124 & 0.393092 & 6 \\
\hline MOL004805 & $\begin{array}{l}\text { (2S)-2-[4-hydroxy-3-(3-methylbut-2-enyl) phenyl]-8,8-dimethyl-2,3- } \\
\text { dihydropyrano[2,3-f]chromen-4-one }\end{array}$ & 2.635983 & $8.38 \mathrm{E}-05$ & 0.379365 & 6 \\
\hline MOL004829 & Glepidotin B & 2.552301 & 4.00E-04 & 0.391803 & 6 \\
\hline MOL004855 & Licoricone & 2.610879 & $2.78 \mathrm{E}-04$ & 0.383013 & 6 \\
\hline MOL004910 & Glabranin & 2.552301 & $3.65 \mathrm{E}-04$ & 0.391803 & 6 \\
\hline MOL004913 & 1,3-dihydroxy-9-methoxy-6-benzofurano[3,2-c]chromenone & 2.569038 & $1.41 \mathrm{E}-04$ & 0.389251 & 6 \\
\hline MOL000239 & Jaranol & 2.560669 & $1.84 \mathrm{E}-04$ & 0.390523 & 5 \\
\hline MOL004077 & sugeonyl acetate & 3.263598 & 4.74E-04 & 0.30641 & 5 \\
\hline MOL004598 & 3,5,6,7-tetramethoxy-2-(3,4,5-trimethoxyphenyl)chromone & 2.619247 & $7.81 \mathrm{E}-04$ & 0.381789 & 5 \\
\hline MOL004609 & Areapillin & 2.552301 & 8.83E-04 & 0.391803 & 5 \\
\hline MOL004624 & Longikaurin A & 3.230126 & $5.23 \mathrm{E}-04$ & 0.309585 & 5 \\
\hline MOL004806 & euchrenone & 2.661088 & 0.001185 & 0.375786 & 5 \\
\hline MOL004838 & 8-(6-hydroxy-2-benzofuranyl)-2,2-dimethyl-5-chromenol & 2.585774 & $2.14 \mathrm{E}-04$ & 0.386731 & 5 \\
\hline MOL004866 & 2-(3,4-dihydroxyphenyl)-5,7-dihydroxy-6-(3-methylbut-2-enyl)chromone & 2.543933 & $2.88 \mathrm{E}-04$ & 0.393092 & 5 \\
\hline MOL004882 & Licocoumarone & 2.577406 & $1.09 \mathrm{E}-04$ & 0.387987 & 5 \\
\hline MOL004898 & $\begin{array}{l}\text { (E)-3-[3,4-dihydroxy-5-(3-methylbut-2-enyl)phenyl]-1-(2,4- } \\
\text { dihydroxyphenyl)prop-2-en-1-one }\end{array}$ & 2.577406 & $1.00 \mathrm{E}-04$ & 0.387987 & 5 \\
\hline MOL004914 & 1,3-dihydroxy-8,9-dimethoxy-6-benzofurano[3,2-c]chromenone & 2.577406 & $1.00 \mathrm{E}-04$ & 0.387987 & 5 \\
\hline MOL000492 & $(+)$-catechin & 2.560669 & 0.001843 & 0.390523 & 4 \\
\hline MOL002341 & Hesperetin & 2.711297 & $6.85 \mathrm{E}-04$ & 0.368827 & 4 \\
\hline MOL004058 & Khell & 2.594142 & $5.80 \mathrm{E}-04$ & 0.385484 & 4 \\
\hline MOL004935 & Sigmoidin-B & 2.594142 & 1.39E-04 & 0.385484 & 4 \\
\hline MOL004948 & Isoglycyrol & 2.661088 & 3.77E-05 & 0.375786 & 4 \\
\hline MOL004989 & 6-prenylated eriodictyol & 2.594142 & $6.51 \mathrm{E}-05$ & 0.385484 & 4 \\
\hline MOL005001 & Gancaonin $\mathrm{H}$ & 2.594142 & 1.39E-04 & 0.385484 & 4 \\
\hline MOL005018 & Xambioona & 2.669456 & 3.34E-05 & 0.374608 & 4 \\
\hline MOL005100 & 5,7-dihydroxy-2-(3-hydroxy-4-methoxyphenyl)chroman-4-one & 2.711297 & $9.02 \mathrm{E}-04$ & 0.368827 & 4 \\
\hline MOL013187 & Cubebin & 2.661088 & $5.88 \mathrm{E}-04$ & 0.375786 & 4 \\
\hline MOL000433 & FA & 2.820084 & $9.38 \mathrm{E}-04$ & 0.354599 & 3 \\
\hline MOL001924 & paeoniflorin & 3.39749 & $2.70 \mathrm{E}-04$ & 0.294335 & 3 \\
\hline MOL002157 & wallichilide & 3.39749 & $5.56 \mathrm{E}-04$ & 0.294335 & 3 \\
\hline MOL004903 & liquiritin & 2.736402 & $3.02 \mathrm{E}-04$ & 0.365443 & 3 \\
\hline MOL004988 & Kanzonol F & 2.686192 & $1.86 \mathrm{E}-05$ & 0.372274 & 3 \\
\hline MOL004993 & 8-prenylated eriodictyol & 2.60251 & $4.00 \mathrm{E}-05$ & 0.384244 & 3 \\
\hline MOL010489 & Resivit & 2.702929 & 2.67E-04 & 0.369969 & 3 \\
\hline MOL001494 & Mandenol & 2.962343 & $6.90 \mathrm{E}-04$ & 0.337571 & 2 \\
\hline MOL001645 & Linoleyl acetate & 2.953975 & $1.51 \mathrm{E}-04$ & 0.338527 & 2 \\
\hline
\end{tabular}

Page $12 / 25$ 


\begin{tabular}{|c|c|c|c|c|c|}
\hline MOL ID & Name & $\begin{array}{l}\text { Average } \\
\text { Shortest } \\
\text { Path } \\
\text { Length }\end{array}$ & $\begin{array}{l}\text { Betweenness } \\
\text { Centrality }\end{array}$ & $\begin{array}{l}\text { Closeness } \\
\text { Centrality }\end{array}$ & Degree \\
\hline MOL001918 & paeoniflorgenone & 3.330544 & 1.14E-04 & 0.300251 & 2 \\
\hline MOL001919 & $\begin{array}{l}\text { (3S,5R,8R,9R,10S,14S)-3,17-dihydroxy-4,4,8,10,14-pentamethyl-2,3,5,6,7,9- } \\
\text { hexahydro-1H-cyclopenta[a]phenanthrene-15,16-dione }\end{array}$ & 3.389121 & 1.35E-04 & 0.295062 & 2 \\
\hline MOL002140 & Perlolyrine & 3.112971 & 4.34E-04 & 0.321237 & 2 \\
\hline MOL004068 & rosenonolactone & 3.330544 & $1.00 \mathrm{E}-04$ & 0.300251 & 2 \\
\hline MOL004653 & (+)-Anomalin & 3.087866 & 1.27E-04 & 0.323848 & 2 \\
\hline MOL004718 & a-spinasterol & 3.355649 & $1.74 \mathrm{E}-04$ & 0.298005 & 2 \\
\hline MOL004924 & (-)-Medicocarpin & 2.753138 & 2.33E-05 & 0.363222 & 2 \\
\hline MOL005815 & Citromitin & 2.803347 & 3.50E-04 & 0.356716 & 2 \\
\hline MOL013381 & Marmin & 3.154812 & 1.47E-04 & 0.316976 & 2 \\
\hline
\end{tabular}

\subsection{Enrichment analysis of GO and KEGG pathway}

We enriched the BP, MF, CC of GO analysis and KEGG pathway enrichment on the common drug-disease targets. By citing the String database, the items with the corrected $\mathrm{P}<0.05$ were screened, and a total of 1850 biological processes were enriched, which included reactions to drugs, reactions to metal ions, reactions to lipopolysaccharides, reactions to bacteria-derived molecules, tube diameter adjustment, blood vessel diameter adjustment, tube diameter adjustment, vascular processes in the circulatory system, response to alcohol, cell response to chemical stress, response to oxidative stress, and so on. They involved 155 molecular functions related to membrane rafts, membrane microzones, membrane regions, postsynaptic membranes, components of postsynaptic membranes, internal components of postsynaptic membranes, synaptic membranes, components of synaptic membranes, internal components of synaptic membranes, presynaptic membrane membrane membrane components, etc. They related 74 cell components, such as neurotransmitter receptor activity, postsynaptic neurotransmitter receptor activity, $\mathrm{G}$ protein-coupled amine receptor activity, catecholamine binding, drug binding, and participation in postsynaptic regulation. Neurotransmitter receptor activity of membrane potential, nuclear receptor activity, ligand-activated transcription factor activity, emitter-gated ion channel activity, emitter-gated channel activity, etc. Excluding tuberculosis, whooping cough, malaria, and measles, we enriched 149 signaling pathways, which mainly included lipids and atherosclerosis, AGE-RAGE signaling pathway in diabetic complications, fluid shear stress and atherosclerosis, Kaposi's sarcoma-associated herpes virus infection, neuroactive ligand-receptor interaction, IL-17 signaling pathway, Chagas disease, TNF signaling pathway, toxoplasmosis, endocrine resistance, chemical carcinogenesis-receptor activation, Th17 cell differentiation, dopaminergic synapses, hepatitis B, Toll-like receptor signaling pathway, osteoclast differentiation, nonalcoholic fatty liver and other signaling pathways related to inflammation, immunity, and oxidative stress. After installing and citing the clusterProfiler package of R 4.0.3, we drew a histogram and bubble chart to visualize the top 20 items with the highest saliency, as shown in Fig. 6A, 6B, 6C and 6D.

\subsection{Construction component-disease-pathway-target network and chord diagram visualization}

We imported the component-disease-pathway-target network file into Cytoscape 3.8.0 to draw the path network diagram. More intuitively, the characteristics of the multicomponent-multitarget effect of the TCM active ingredients in the treatment of diseases were revealed, as shown in Fig. 7 (blue is the compound, yellow is the target of the Chinese medicine on the disease, green is the top 20 most significant pathways, red is the disease that is the disease, and purple is the Chinese medicine). The KEGG signaling pathway of drug-disease was visually analyzed using the Sangerbox tool, and it was found that the top 20 signaling pathways significantly enriched by KEGG involved 85 genes, as shown in Fig. 8 .

\subsection{Molecular docking}

We used Pymol2.1 software to visualize the complex formed by the compounds and proteins after molecular docking (select the compound with the most negative binding energy score for each target), which obtained the binding mode of the compound and the protein, as shown in Table 4. According to the binding mode, the amino acid residues where the compound binds to the protein pocket could be clearly seen; for example, kaempferol binds to AKT1. The active amino acid residues included VAL-164, GLU-228, ALA-230, ASP-292, MET-281, GLU-234, etc. Kaempferol is a flavonoid compound that contains multiple hydroxyl groups and can interact with the active groups of amino acids to form hydrogen bonds. For example, it could interact with the active groups of GLU-228, ASP-292, ALA-230, and GLU-234. The strong hydrogen bond interaction, with an average hydrogen bond distance of $2.0 \AA$, was much smaller than the traditional hydrogen bond of $3.5 \AA$, which played an important role in stabilizing small molecule ligands. In summary, kaempferol, luteolin, quercetin, and 7-methoxy-2-methyl isoflavone compounds matched well with the five target protein targets, could form stable complexes, and had a good relationship with the protein, which also indirectly verified these compounds. It had a regulatory effect on AKT1, IL 1B, IL-6, FOS, GSK3B, GABRA and other targets. When the binding energy is less than 0 , it is

Page $13 / 25$ 
considered that the ligand and receptor can bind freely, and the lower the binding energy is, the stronger the affinity. These results indicated that the molecular docking results were consistent with the screening results of network pharmacology, which preliminarily verified the reliability of network pharmacology, as shown in Fig. 9.

Table 4. The molecular docking results of selected compounds

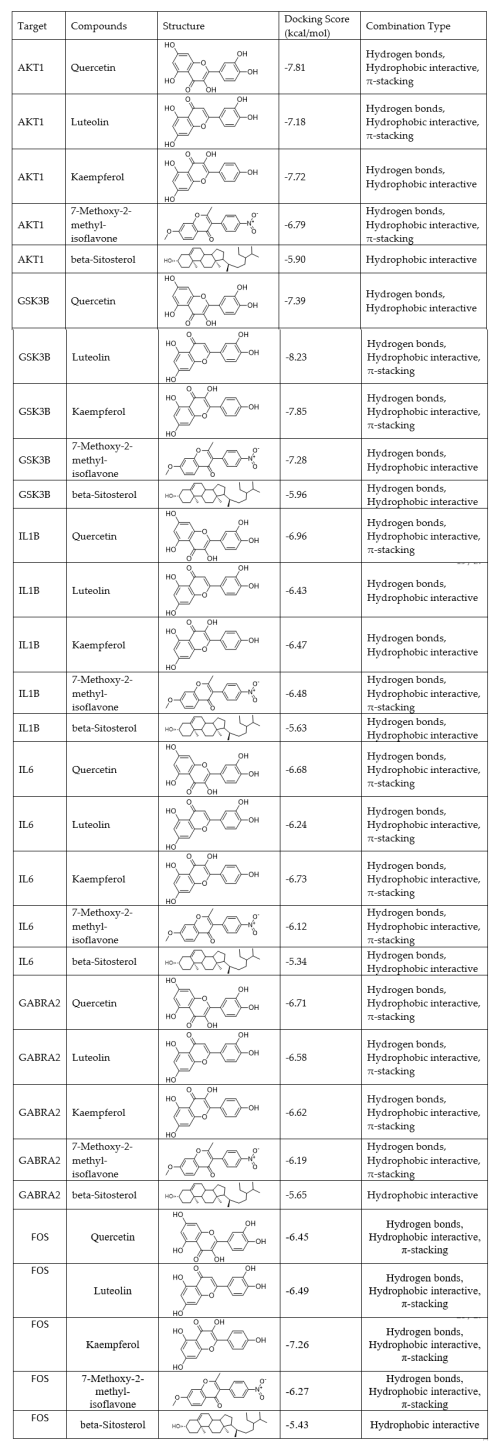

Note: Binding energy fuction [28]:

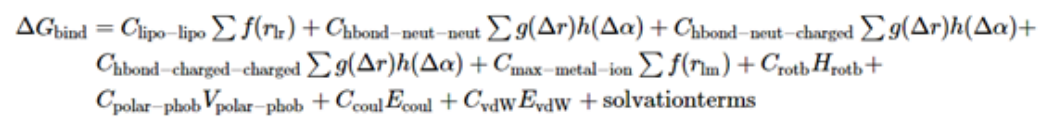

Molecular docking data indicated that the binding capacity of GSK3B and luteolin (E) was significant.

\section{Discussion}

Under the current COVID-19 pandemic, many people with depression and insomnia may be related to environmental mutations and social anxiety. The study found that the symptom levels of depression, anxiety and insomnia in the quarantine area were significantly higher than those in the nonquarantine area, and they were at higher risk of depression, anxiety and insomnia, especially the severity of depression [30]. TCM believes that the main pathogenesis of insomnia is the imbalance of Qi and the inability of Yang to enter Yin. The Yellow Emperor's Internal Classic states, "If you are unable to make decisions, or if your emotions are not smooth, the liver qi will be stagnant, and the qi axis will not turn, but if you want to stretch, you will be inward. Disturb the soul and cause insomnia", and depression is due to stagnation of liver qi, so liver-regulating qi is the basic rule for the treatment of insomnia and depression, suggesting that qi-regulating drugs are potential drugs for the treatment of depression. 
CHSGP is composed of 7 herbs: Chaihu, Xiangfu, Chuanxiong, Chenpi, Zhike, Baishao, and Gancao. Saikosaponin, the main chemical component of Chaihu, has sedative, antipyretic, immune enhancement, anti-inflammatory, antiviral, liver, and antitumor effects. The a-germanone of Xiangfu has an antidepressant-like effect [31], and Jiawei Xiangfu Decoction combined with acupuncture can significantly improve the sleep state of menopausal insomnia patients with liver stagnation and qi stagnation [32]. The main active ingredients of Chuanxiong have both a sedative effect[26] and the potential to improve depression. The antidepressant mechanism of Chenpi and its main active ingredients, chuanchenein, hesperidin and naringenin, is related to the improvement of neurobiochemical, neuroendocrine and neurotrophic systems [33]. Zhike exerts antidepressant effects mainly through its monoaminergic mechanism and prokinetic effects [34]. Fructus aurantii can enhance gastrointestinal motility by changing the expression level of 5-HT in the gastrointestinal tract of rats [35], suggesting that it can affect sleep to a certain extent. Paeoniflorin has neuroprotective and antidepressant biological activities, and it can treat related insomnia diseases through adenosine A1R to play a hypnotic effect [36]. Regulating the calmodulin/calmodulin-dependent protein kinase II (CaM/CaMKII) pathway and its downstream signaling molecules play an important role in the treatment and alleviation of affective disorders [37]. Glycyrrhizin can reduce IL-6 cytokines in brain tissue, and it is also a cytokine closely related to sleep/deprivation. IL-6 can inhibit the synthesis of IL- 1 and reduce IL- 6 cytokines, and then IL- 1 increases synthesis, which in turn enhances the patient's nonrapid eye movement sleep time [38].

The results of the study showed that topological analysis identified the key components of quercetin, kaempferol, $\beta$-sitosterol, 7-methoxy-2-methyl isoflavones, luteolin, naringenin and other key components in CHSGP as the disease. It was the material basis for the treatment of insomnia and depression with the same treatment of different diseases. It had the largest number of targets and was also its core compound. Studies have shown that quercetin enhanced the hypnotic activity of pentobarbital in a dose-dependent manner by prolonging sleep time [39] and reducing anxiety and depression-like behaviors, immune dysfunction and brain oxidative stress [40], related to its improvement of neurotrophic function and antiinflammatory effects[41]. Kaempferol has a wide range of pharmacological activities, which can reduce oxidative stress and proinflammatory cytokines, inhibit vascular endothelial inflammation [42], and have an effective antidepressant effect [26, 43]. Studies have found [44] that some Chinese herbal extracts contain $\beta$-sitosterol, which has anti-inflammatory, sedative and hypnotic activities [45]. Luteolin is a widely used flavonoid compound that has anti-anxiety and sleep-promoting effects [46]. Naringenin can activate Sirt1, enhance antioxidant capacity by reducing oxidative stress, and effectively improve inflammation and dopamine levels [47], thereby alleviating the pain caused by chronic sleep deprivation [48].

According to the drug-disease target intersection, CHSGP can treat insomnia and depression at the same time through 113 target proteins in different diseases. According to the degree value and the betweenness centrality, PPI analysis network topology analysis screened AKT1 and IL-6, and 42 core targets, such as IL1B, CASP3, and MAPK3, were mainly related to immunity, inflammation, oxidative stress, and neovascularization. AKT1 is involved in the regulation of processes such as metabolism, proliferation, growth and angiogenesis [49]. IL-6 is one of the most biologically active cytokines and can be called a sleepy mediator. Its circadian rhythm pattern reflects the steady-state drive of sleep [50], and increased IL-6 activity may cause depression by activating the hypothalamus-pituitary-adrenal axis or affecting neurotransmitter metabolism [51]. IL-1B protein is an important mediator of the inflammatory response, is involved in a variety of cell activities, such as cell proliferation, differentiation, and apoptosis, and is related to the diagnosis, specific symptoms, and antidepressant treatment response of major depression [1]. Caspase-3 (CASP3) belongs to the cysteine aspartic protease (Caspase) family of proteases and plays an irreplaceable role in the apoptosis pathway. Hippocampal apoptosis caused by sleep deprivation includes the number of apoptotic cells, caspase-3 activation, and Bax and Bcl-2 regulation[52]. MAPK3, called mitogen-activated protein kinase 3 , is an important part of the MAP kinase signal transduction pathway and plays an important role in the cellular response cascade caused by extracellular stimulation. Another study[53] showed that the expression of the AKT1, MAPK3 and IL-6 genes in anxiety patients often increased significantly. The MCODE cluster analysis in the PPI network screened 7 core genes, including FOS, GABRA2, GSK3B, PON1, APOB, CYP1B1, and ADRA1A. FOS, also known as c-Fos, is a proto-oncogene that participates in cell growth, differentiation, information transmission, animal learning and memory and maintains wakefulness. Studies have shown that [54] FOS protein is involved in the normal differentiation, growth, learning, memory and other processes of cells, and it is more highly expressed in the brain endothelium, hippocampus, and striatum. GABA is found only in the nervous tissues of animals. It is an important inhibitory neurotransmitter and plays a role in the development process. It participates in a variety of metabolic activities and has high physiological activity. The GABRA2 gene encodes the GABAA-2 subunit, which is an ionotropic receptor related to anxiety, depression, and other behavioral disorders (including drug dependence and schizophrenia). Studies have found that[55] a higher expression level of a2 may have antidepressant effects through the signal transduction of GABAAR containing a2.

PPI network and GO analysis showed that the strong interactions of these target genes affected the synaptic membrane and postsynaptic membrane by participating in biological processes such as drug response, regulation of blood vessel diameter, and oxidative stress response, which included the molecular functions of synaptic membrane, postsynaptic membrane and its components, and which involved cell functions such as neurotransmitter receptor activity, postsynaptic neurotransmitter receptor activity, G protein coupled amine receptor activity, etc. The KEGG pathway enrichment analysis mainly involved the AGE-RAGE signaling pathway, neuroactive ligand-receptor interaction, IL-17 signaling pathway, TNF signaling pathway, endocrine resistance, Th17 cell differentiation, dopaminergic synapses, hepatitis B, Toll-like receptor signaling pathways and other signaling pathways related to inflammation, immunity, and oxidative stress.

Studies have found that the inhibition of the AGE/RAGE pathway and oxidative stress might be a possible mechanism by which melatonin exerts antidepressant and anti-anxiety effects in diabetic rats[56]. AGE-RAGE signaling is among the signaling pathways related to obstructive sleep apnea (OSA)[57]. A number of network pharmacology studies have found that TCM has neuroactive ligand-receptor interaction pathways in the treatment

Page $15 / 25$ 
of depression or insomnia [16,58-60]. A literature study found that it might have a beneficial effect on the pathogenic conditions of the central nervous system by inhibiting the function of the IL-17 cytokine family [61].

The molecular docking results showed that the molecular docking in compounds (kaempferol, luteolin, quercetin, 7-methoxy-2-methyl isoflavone and beta-sitosterol) and five target proteins (AKT1, IL1B, IL-6, FOS, GSK3B, and GABRA) matched well, and the binding energy was less than - 6 kcal/mol, which could form stable complexes and indirectly verified that these compounds had a regulatory effect on targets such as AKT1.

This study is aimed at the high incidence of global depression and insomnia under the current situation of the COVID-19 epidemic, and it is difficult to find effective prevention methods in clinical practice. At the same time, the network pharmacology of CHSGP provides us with an opportunity to explore the "Same Treatment for Different Diseases" potential pharmacological mechanism of CHSGP in treating insomnia and depression caused by the COVID-19 epidemic. Existing network pharmacology studies have screened 121 active ingredients and 15 depression-related targets of CHSGP from the database, mainly involving the regulation of neurotransmitters (serotonin, dopamine and adrenaline), the regulation of inflammatory mediators of TRP channels, calcium signaling pathway, cyclic adenosine monophosphate signaling pathway, and neural active ligand-receptor interaction, which are the channels through which signals play an antidepressant effect. It can have an antidepressant effect by improving neuronal plasticity, growth, transfer conditions and gene expression in neuronal cells and increasing the expression of gap junction proteins [16]. Animal experiments [62] confirmed that CHSGP can improve the depression-like behavior of rats exposed to chronic unpredictable mild stress (CUMS), possibly by inhibiting CHOP and caspase-12-mediated apoptosis of rat hippocampal cells. CHSGP can significantly improve the depression state of model rats by increasing the expression of BDNF and TrkB mRNA in the hippocampus, amygdala and frontal lobe [63]. A large number of clinical studies [64-66] have shown that CHSGP alone or combined with Western medicine has a significant effect on the treatment of insomnia, and network pharmacology data mining CHSGP may play a role in the treatment of insomnia through multiple components, multiple targets, and multiple pathways [67].

Although the research has some findings, it still has many limitations. First, due to incomplete information, the role of some CHSGP compounds in "Same Treatment for Different Diseases" may be ignored. Second, it is difficult to screen specific targets and key active ingredients directly from the complex cognitive CHSGP. Third, although CHSGP has antidepressant and sleep-improving effects in previous clinical studies and animal model experiments, its mechanism of action is still unclear, and further analysis and excavation are needed in subsequent clinical and animal experiments. Therefore, it is necessary to further study the role and specific targets and pathways of the important single components of the CHSGP in the process of treating insomnia and depression with the "Same Treatment for Different Diseases".

\section{Conclusions}

In summary, this study used network pharmacology to find that the mechanism of CHSGP for insomnia and depression caused by the COVID-19 epidemic with "Same Treatment for Different Diseases" might be related to core targets such as AKT1, IL-6, IL1B, FOS, CASP3, MAPK3, and KEGG signaling pathways such as the AGE-RAGE pathway, neuroactive ligand-receptor interaction, and IL-17 signaling pathway. The results of molecular docking revealed that kaempferol, luteolin, quercetin and other compounds matched well with AKT1, IL1B, IL-6, FOS, GSK3B, GABRA and other target protein molecules. The overall analysis and verification of the relationship among CHSGP, insomnia and depression were the "Same Treatment for Different Diseases" relationship. In molecular biology research, in-depth pharmacological research and clinical application exploration of insomnia and depression provide a reference. However, this research is not perfect enough. It is necessary to carry out relevant clinical and animal experimental studies in the complex environmental background of the COVID-19 epidemic to verify its clinical efficacy and mechanism of action of CHSGP for insomnia and depression with the "Same Treatment for Different Diseases".

\section{Declarations}

\section{Acknowledgements}

We are grateful to the National Natural Science Foundation of China and the Qi Huang Scholars Program for funding. Thanks to Li Shaodan, Yang Minghui and other authors for their help and efforts in the process of conception, writing, revision and submission. We particularly thank Dr. Ruojun Zhang (Beijing Institute of Technology) for the English translation of the dissertation.

\section{Author contributors}

Conceptualization, L.W. and P.W.; methodology, L.W. and P.W.; investigation, L.W., Y.C., and C.L.; writing-original draft preparation,L.W.; writingreview and editing, L.W., X.W., and M.L.; visualization, L.W., Z.L. and Y.Z.; funding acquisition, S.L. and M.Y. All authors have read and agreed to the published version of the manuscript.

\section{Funding}

This study was supported by the Inheritance and Innovation of TCM "Hundreds, Thousands, Tens of Thousands" Talent Project Qihuang Scholars Fund ([2018]12) and National Natural Science Foundation of China (N0.82174283). 
Availability of data and materials

All data generated or analyzed during this study are included in this published article.

\section{Ethics approval and consent to participate}

Not applicable.

\section{Consent for publication}

Not applicable.

\section{Competing interests}

The authors declare that they have no competing interests.

\section{References}

1. Sacks D, Baxter B, Campbell B, et al. Multisociety Consensus Quality Improvement Revised Consensus Statement for Endovascular Therapy of Acute Ischemic Stroke[J]. Int J Stroke, 2018, 13(6):612-632. DOI: 10.1177/1747493018778713.

2. Marvaldi M, Mallet J, Dubertret C, et al. Anxiety, depression, trauma-related, and sleep disorders among healthcare workers during the COVID-19 pandemic: A systematic review and meta-analysis[J]. Neurosci Biobehav Rev, 2021, 126:252-264. DOI: 10.1016/j.neubiorev.2021.03.024.

3. Lang Q, Liu X, He Y, et al. Association between Working Hours and Anxiety/Depression of Medical Staff during Large-Scale Epidemic Outbreak of COVID-19: A Cross-Sectional Study[J]. Psychiatry Investig, 2020, 17(12):1167-1174. DOI: 10.30773/pi.2020.0229.

4. Huang Y, Zhou Y, Wei Y, et al. Differences in the Association of Anxiety, Insomnia and Somatic Symptoms between Medical Staff and the General Population During the Outbreak of COVID-19[J]. Neuropsychiatr Dis Treat, 2021, 17:1907-1915. DOI: 10.2147/NDT.S300719.

5. Zhang Z, Hu Y, Chen Y, et al. Sleep disorders and related factors among frontline medical staff supporting Wuhan during the COVID-19 outbreak[J]. Bull Menninger Clin, 2021 :1-17. DOI: 10.1521/bumc_2021_85_01.

6. Morin CM, Drake CL, Harvey AG, et al. Insomnia disorder[J]. Nat Rev Dis Primers, 2015, 1:15026. DOI: 10.1038/nrdp.2015.26.

7. Wang YS, Shen CY, Jiang JG. Antidepressant active ingredients from herbs and nutraceuticals used in TCM: pharmacological mechanisms and prospects for drug discovery[J]. Pharmacol Res, 2019, 150:104520. DOI: 10.1016/j.phrs.2019.104520.

8. Asarnow LD, Manber R. Cognitive Behavioral Therapy for Insomnia in Depression[J]. Sleep Med Clin, 2019, 14(2):177-184. DOI: 10.1016/j.jsmc.2019.01.009.

9. Liu L, Liu C, Wang Y, et al. Herbal Medicine for Anxiety, Depression and Insomnia[J]. Curr Neuropharmacol, 2015, 13(4):481-493. DOI: $10.2174 / 1570159 \times 1304150831122734$.

10. Riemann D, Krone LB, Wulff K, et al. Sleep, insomnia, and depression[J]. Neuropsychopharmacology, 2020, 45(1):74-89. DOI: 10.1038/s41386019-0411-y.

11. Morin CM, Bjorvatn B, Chung F, et al. Insomnia, anxiety, and depression during the COVID-19 pandemic: an international collaborative study[J]. Sleep Med, 2021, 87:38-45. DOI: 10.1016/j.sleep.2021.07.035.

12. Unützer J, Klap R, Sturm R, et al. Mental disorders and the use of alternative medicine: results from a national survey[J]. Am J Psychiatry, 2000, 157(11):1851-1857. DOI: 10.1176/appi.ajp.157.11.1851.

13. Liu P, Yang P, Zhang L. Mode of Action of Shan-Zhu-Yu (Cornus officinalis Sieb. et Zucc.) in the Treatment of Depression Based on Network Pharmacology[J]. Evid Based Complement Alternat Med, 2020, 2020:8838888. DOI: 10.1155/2020/8838888.

14. Zhang YW, Cheng YC. Challenge and Prospect of Traditional Chinese Medicine in Depression Treatment[J]. Front Neurosci, 2019, 13:190. DOI: 10.3389/fnins.2019.00190.

15. Tang XS, Chen J, Fang G, et al. Discussion on the mechanism of Chaihu Shugan Powder in the treatment of insomnia based on network pharmacology[J]. Chinese Modern Chinese Medicine, 2021, 23(06):1013-1022.

16. Liu YY, Hu D, Fan QQ, et al. Mechanism of Chaihu Shugan Powder () for Treating Depression Based on Network Pharmacology[J]. Chin J Integr Med, 2020, 26(12):921-928. DOI: 10.1007/s11655-019-3172-x.

17. Xiao K, Li K, Long S, et al. Potential Molecular Mechanisms of Chaihu-Shugan-San in Treatment of Breast Cancer Based on Network Pharmacology[J]. Evid Based Complement Alternat Med, 2020, 2020:3670309. DOI: 10.1155/2020/3670309.

18. Guo H, Zhao CY, Zhan LB, et al. The mechanism of Liuwei Dihuang Pill in the treatment of hypertension, type 2 diabetes mellitus and Alzheimer's disease based on network pharmacology[J]. Pharmacology and Clinic of Traditional Chinese Medicine, 2021, 37(01):41-49.

19. Huang MW, Yang HJ, Zhou XC, et al. [Advances on network pharmacology in ethnomedicine research][J]. Zhongguo Zhong Yao Za Zhi, 2019, 44(15):3187-3194. DOI: 10.19540/j.cnki.cjcmm.20190711.201. 
20. Luo TT, Lu Y, Yan SK, et al. Network Pharmacology in Research of Chinese Medicine Formula: Methodology, Application and Prospective[J]. Chin J Integr Med, 2020, 26(1):72-80. DOI: 10.1007/s11655-019-3064-0.

21. Ru J, Li P, Wang J, et al. TCMSP: a database of systems pharmacology for drug discovery from herbal medicines[J]. J Cheminform, $2014,6: 13$. DOI: $10.1186 / 1758-2946-6-13$.

22. UniProt: a worldwide hub of protein knowledge[J]. Nucleic Acids Res, 2019, 47(D1):D506-D515. D0I: 10.1093/nar/gky1049.

23. Szklarczyk D, Gable AL, Lyon D, et al. STRING v11: protein-protein association networks with increased coverage, supporting functional discovery in genome-wide experimental datasets[J]. Nucleic Acids Res, 2019, 47(D1):D607-D613. D0I: 10.1093/nar/gky1131.

24. Sun C, Yuan Q, Wu D, et al. Identification of core genes and outcome in gastric cancer using bioinformatics analysis[J]. Oncotarget, 2017, 8(41):70271-70280. DOI: 10.18632/oncotarget.20082.

25. Morris GM, Lim-Wilby M. Molecular docking[J]. Methods Mol Biol, 2008, 443:365-382. DOI: 10.1007/978-1-59745-177-2_19.

26. Rajeswari M, Santhi N, Bhuvaneswari V. Pharmacophore and Virtual Screening of JAK3 inhibitors[J]. Bioinformation, 2014, 10(3):157-163. DOI: $10.6026 / 97320630010157$.

27. Fazi R, Tintori C, Brai A, et al. Homology Model-Based Virtual Screening for the Identification of Human Helicase DDX3 Inhibitors[J]. J Chem Inf Model, 2015, 55(11):2443-2454. DOI: 10.1021/acs.jcim.5b00419.

28. Friesner RA, Banks JL, Murphy RB, et al. Glide: a new approach for rapid, accurate docking and scoring. 1. Method and assessment of docking accuracy[J]. J Med Chem, 2004, 47(7):1739-1749. DOI: 10.1021/jm0306430.

29. Doncheva NT, Morris JH, Gorodkin J, et al. Cytoscape StringApp: Network Analysis and Visualization of Proteomics Data[J]. J Proteome Res, 2019, 18(2):623-632. DOI: 10.1021/acs.jproteome.8b00702.

30. Wang C, Song W, Hu X, et al. Depressive, anxiety, and insomnia symptoms between population in quarantine and general population during the COVID-19 pandemic: a case-controlled study[J]. BMC Psychiatry, 2021, 21(1):99. DOI: 10.1186/s12888-021-03108-2.

31. Xia B, Tong Y, Xia C, et al. a-Cyperone Confers Antidepressant-Like Effects in Mice via Neuroplasticity Enhancement by SIRT3/ROS Mediated NLRP3 Inflammasome Deactivation[J]. Front Pharmacol, 2020, 11:577062. DOI: 10.3389/fphar.2020.577062.

32. Yan XL, Yu YD, Yang DD. [Clinical efficacy of acupuncture combined with modified Xiangfu Decoction in treatment of menopausal insomnia cause by liver Qi stagnation][J]. Zhongguo Zhong Yao Za Zhi, 2020, 45(6):1460-1464. DOI: 10.19540/j.cnki.cjcmm.20191010.501.

33. Bansal Y, Singh R, Saroj P, et al. Naringenin protects against oxido-inflammatory aberrations and altered tryptophan metabolism in olfactory bulbectomized-mice model of depression[J]. Toxicol Appl Pharmacol, 2018, 355:257-268. DOI: 10.1016/j.taap.2018.07.010.

34. Zhang YJ, Huang W, Huang X, et al. Fructus Aurantii induced antidepressant effect via its monoaminergic mechanism and prokinetic action in rat[J]. Phytomedicine, 2012, 19(12):1101-1107. DOI: 10.1016/j.phymed.2012.05.015.

35. Jiang Y, Bai X, Zhu X, et al. The effects of Fructus Aurantii extract on the 5-hydroxytryptamine and vasoactive intestinal peptide contents of the rat gastrointestinal tract[J]. Pharm Biol, 2014, 52(5):581-585. DOI: 10.3109/13880209.2013.854396.

36. Yin D, Liu YY, Wang TX, et al. Paeoniflorin exerts analgesic and hypnotic effects via adenosine A1 receptors in a mouse neuropathic pain model[J]. Psychopharmacology (Berl), 2016, 233(2):281-293. DOI: 10.1007/s00213-015-4108-6.

37. Song C, Wang J, Gao D, et al. Paeoniflorin, the Main Active Ingredient of Shuyu Capsule, Inhibits Ca(v)1.2 and Regulates Calmodulin/Calmodulin-Dependent Protein Kinase II Signalling[J]. Biomed Res Int, 2017, 2017:8459287. DOI: 10.1155/2017/8459287.

38. Huang Q, Wang YN, Han F, et al. Study on the potential mechanism of anti-insomnia effect of Guizhi Gancao Decoction based on network pharmacology[J]. Journal of Hunan University of Traditional Chinese Medicine, 2020, 40(04):452-459.

39. Ye MF, Liu Z, Lou SF, et al. Flos Albiziae aqueous extract and its active constituent quercetin potentiate the hypnotic effect of pentobarbital via the serotonergic system[J]. Biomed Rep, 2015, 3(6):835-838. DOI: 10.3892/br.2015.518.

40. Merzoug S, Toumi ML, Tahraoui A. Quercetin mitigates Adriamycin-induced anxiety- and depression-like behaviors, immune dysfunction, and brain oxidative stress in rats[J]. Naunyn Schmiedebergs Arch Pharmacol, 2014, 387(10):921-933. D0I: 10.1007/s00210-014-1008-y.

41. Fang K, Li HR, Chen XX, et al. Quercetin Alleviates LPS-Induced Depression-Like Behavior in Rats via Regulating BDNF-Related Imbalance of Copine 6 and TREM1/2 in the Hippocampus and PFC[J]. Front Pharmacol, 2019, 10:1544. DOI: 10.3389/fphar.2019.01544.

42. Ren J, Lu Y, Qian Y, et al. Recent progress regarding kaempferol for the treatment of various diseases[J]. Exp Ther Med, 2019, 18(4):2759-2776. DOI: 10.3892/etm.2019.7886.

43. Gao W, Wang W, Peng Y, et al. Antidepressive effects of kaempferol mediated by reduction of oxidative stress, proinflammatory cytokines and up-regulation of AKT/ß-catenin cascade[J]. Metab Brain Dis, 2019, 34(2):485-494. DOI: 10.1007/s11011-019-0389-5.

44. Uddin G, Rauf A, Siddiqui BS, et al. Anti-nociceptive, anti-inflammatory and sedative activities of the extracts and chemical constituents of Diospyros lotus L[J]. Phytomedicine, 2014, 21(7):954-959. DOI: 10.1016/j.phymed.2014.03.001.

45. Liu WL, Wu BF, Shang JH, et al. Moringa oleifera Lam Seed Oil Augments Pentobarbital-Induced Sleeping Behaviors in Mice via GABAergic Systems[J]. J Agric Food Chem, 2020, 68(10):3149-3162. DOI: 10.1021/acs.jafc.0c00037.

46. Kim TH, Custodio RJ, Cheong JH, et al. Sleep Promoting Effect of Luteolin in Mice via Adenosine A1 and A2A Receptors[J]. Biomol Ther (Seoul), 2019, 27(6):584-590. DOI: 10.4062/biomolther.2019.149. 
47. Olufunke D, Edidiong A, Oluwatomisin F, et al. Therapeutic activities of naringenin on efavirenz-induced sleep-like disorder in the midbrain of white albino mice[J]. Iran J Basic Med Sci, 2020, 23(11):1462-1470. DOI: 10.22038/ijbms.2020.47043.10852.

48. Arora S, Venugopalan A, Dharavath RN, et al. Naringenin Ameliorates Chronic Sleep Deprivation-Induced Pain via Sirtuin1 Inhibition[J]. Neurochem Res, 2021, 46(5):1177-1187. DOI: 10.1007/s11064-021-03254-9.

49. Palumbo S, Paterson C, Yang F, et al. PKBß/AKT2 deficiency impacts brain mTOR signaling, prefrontal cortical physiology, hippocampal plasticity and select murine behaviors[J]. Mol Psychiatry, 2021, 26(2):411-428. DOI: 10.1038/s41380-020-00964-4.

50. Vgontzas AN, Bixler EO, Lin HM, et al. IL-6 and its circadian secretion in humans[J]. Neuroimmunomodulation, 2005, 12(3):131-140. DOI: 10.1159/000084844.

51. Ting EY, Yang AC, Tsai SJ. Role of Interleukin-6 in Depressive Disorder[J]. Int J Mol Sci, 2020, 21(6). DOI: 10.3390/ijms21062194.

52. Zuo JX, Li M, Jiang L, et al. Hydrogen Sulfide Prevents Sleep Deprivation-Induced Hippocampal Damage by Upregulation of Sirt1 in the Hippocampus[J]. Front Neurosci, 2020, 14:169. DOI: 10.3389/fnins.2020.00169.

53. Liu FX, Lin ZX, Zhang HL, et al. Analysis of the mechanism of Jiawei Guipi Decoction in the treatment of Yin-fire insomnia with anxiety based on network pharmacology[J]. Chinese Journal of Experimental Formulas, 2020, 26(20):161-168.

54. Wang TX, Wei HH, Chen ZK, et al. Hypnotic activities of Zao Ren An Shen capsule, a traditional Chinese medicine, in an anxiety-like mouse model[J]. Sleep Breath, 2020 . DOI: 10.1007/s11325-020-02244-5.

55. Benham RS, Hewage NB, Suckow RF, et al. Prodepressant- and anxiogenic-like effects of serotonin-selective, but not noradrenaline-selective, antidepressant agents in mice lacking a2-containing GABA(A) receptors[J]. Behav Brain Res, 2017, 332:172-179. DOI: 10.1016/j.bbr.2017.05.063.

56. Ergenc M, Ozacmak HS, Turan I, et al. Melatonin reverses depressive and anxiety like-behaviours induced by diabetes: involvement of oxidative stress, age, rage and S100B levels in the hippocampus and prefrontal cortex of rats[J]. Arch Physiol Biochem, 2019 :1-9. DOI: 10.1080/13813455.2019.1684954.

57. Jagannathan R, Seixas A, St-Jules D, et al. Systems Biology Genetic Approach Identifies Serotonin Pathway as a Possible Target for Obstructive Sleep Apnea: Results from a Literature Search Review[J]. Sleep Disord, 2017, 2017:6768323. DOI: 10.1155/2017/6768323.

58. Xiong WC, Wu HZ, Xiong YY, et al. Network Pharmacology-based Research of Active Components of Albiziae Flos and Mechanisms of Its Antidepressant Effect[J]. Curr Med Sci, 2020, 40(1):123-129. DOI: 10.1007/s11596-020-2155-7.

59. Jia Y, Zou J, Wang Y, et al. Action mechanism of Roman chamomile in the treatment of anxiety disorder based on network pharmacology[J]. J Food Biochem, 2021, 45(1):e13547. DOI: 10.1111/jfbc.13547.

60. Jin D, Zhang J, Zhang Y, et al. Network pharmacology-based and molecular docking prediction of the active ingredients and mechanism of ZaoRenDiHuang capsules for application in insomnia treatment[J]. Comput Biol Med, 2021, 135:104562. DOI:

10.1016/j.compbiomed.2021.104562.

61. Waisman A, Hauptmann J, Regen T. The role of IL-17 in CNS diseases[J]. Acta Neuropathol, 2015, 129(5):625-637. DOI: 10.1007/s00401-0151402-7.

62. Sun KH, Jin Y, Mei ZG, et al. Antidepressant-Like Effects of Chaihu Shugan Powder on Rats Exposed to Chronic Unpredictable Mild Stress through Inhibition of Endoplasmic Reticulum Stress-Induced Apoptosis[J]. Chin J Integr Med, 2021, 27(5):353-360. DOI: 10.1007/s11655-0203228-y.

63. Deng Y, Zhang CH, Zhang HN. Effects of chaihu shugan powder on the behavior and expressions of BDNF and TrkB in the hippocampus, amygdala, and the frontal lobe in rat model of depression [J]. Zhongguo Zhong Xi Yi Jie He Za Zhi, 2011, 31(10):1373-1378.

64. LIN C; , LI YH. Clinical observation on the treatment of insomnia after mild craniocerebral trauma with Chaihu Shugan San modified formula [J]. Journal of Guangzhou University of Traditional Chinese Medicine, 2021, 38(05):890-894.

65. Yu M, Wang YC, Li L, et al. Clinical effect of abdominal acupuncture combined with Chaihu Shugan powder in the treatment of perimenopausal insomnia due to qi stagnation [J]. China Medical Herald, 2020, 17(20):160-163.

66. Zhang HZ, Zhu XD, Zhang JX, et al. Observation on the curative effect of Chaihu Shugan Powder combined with Eszopiclone Tablets on insomnia due to liver stagnation and qi stagnation [J]. TCM Research, 2016, 29(06):5-7.

67. Yang DF, Cao LP. Clinical observation on the treatment of insomnia due to liver stagnation with modified Chaihu Shugan Powder [J]. Western Traditional Chinese Medicine, 2015, 28(11):82-84.

\section{Figures}




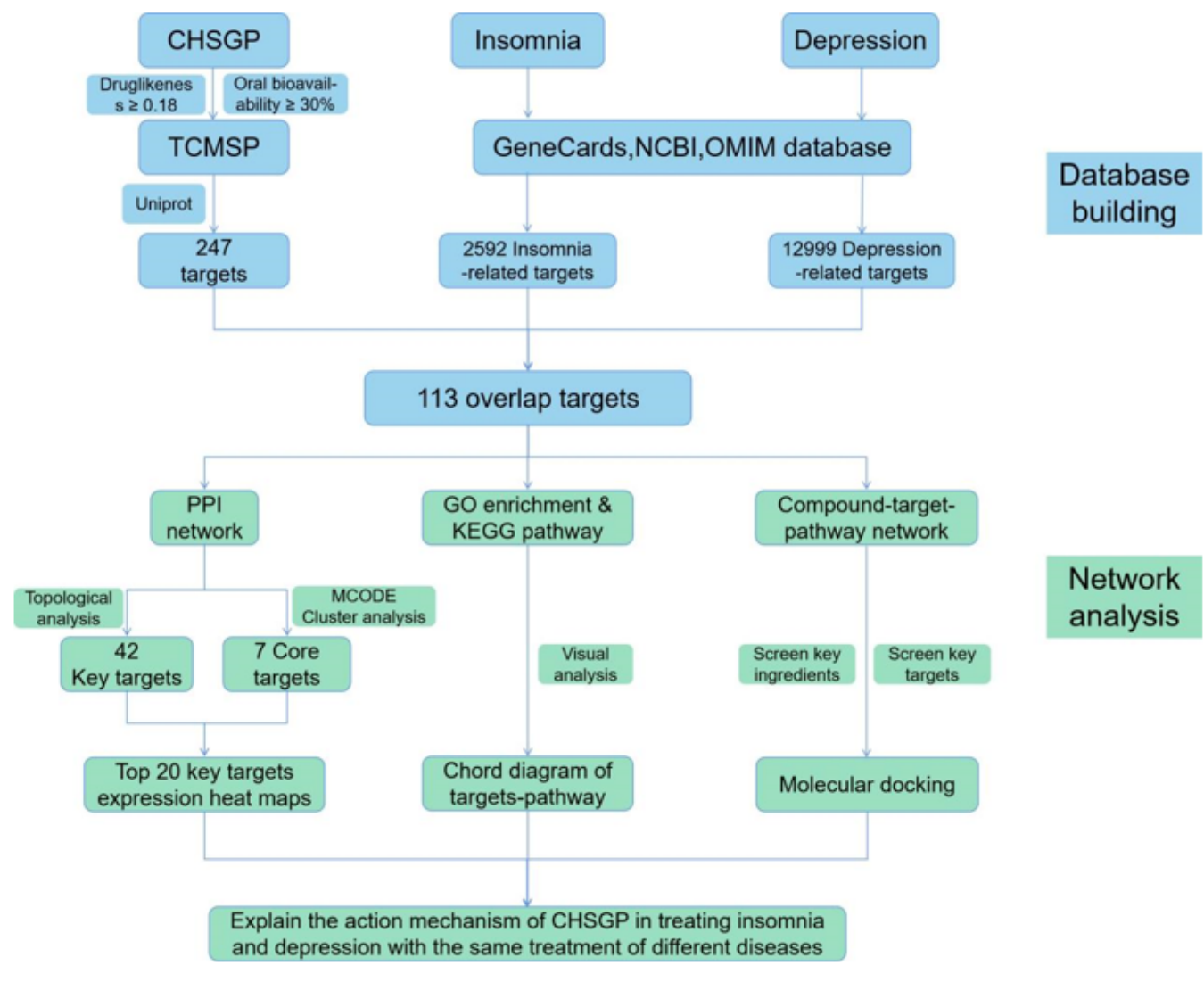

Figure 1

The experimental flow of this study.

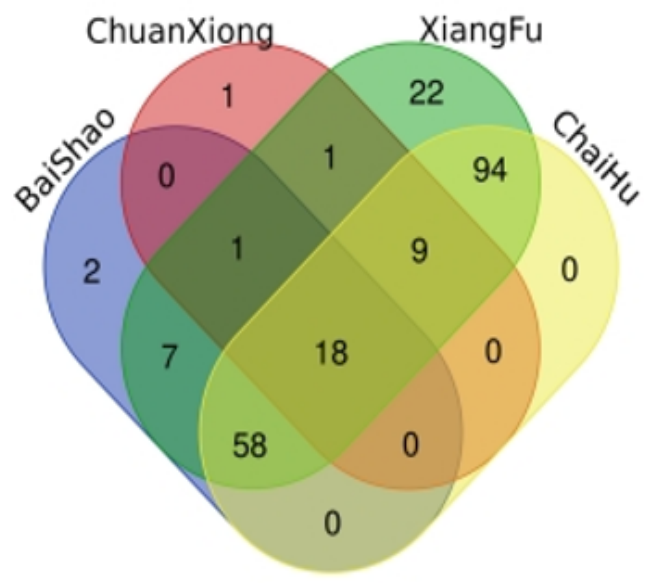

A.Liver Channel

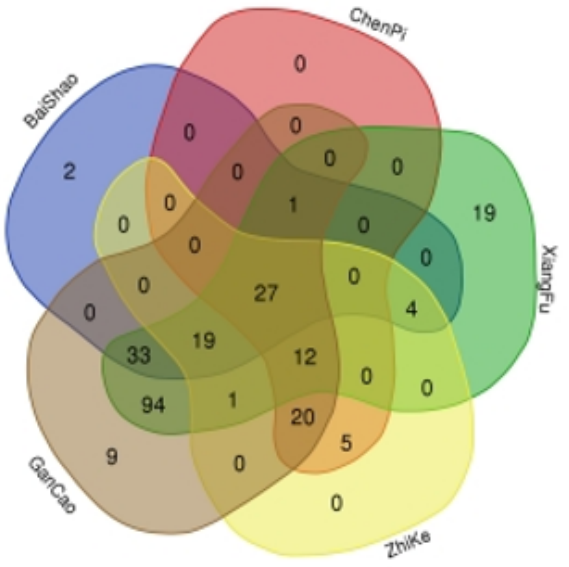

B.Spleen Channel

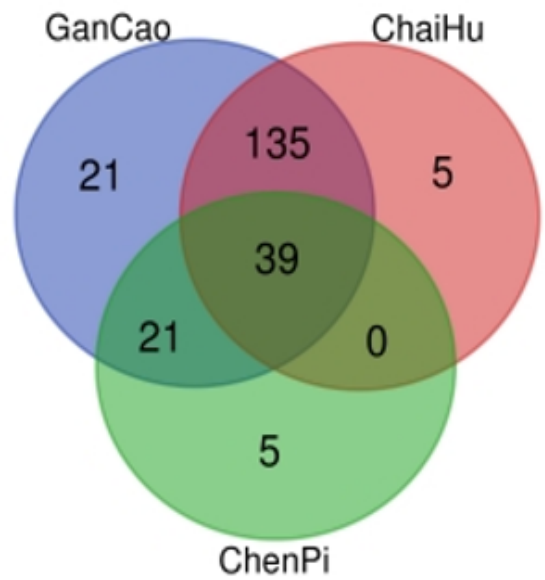

C.Lung Channel

Figure 2

Guijing analysis of each Chinese medicine in CHSGP 


\section{Depression}

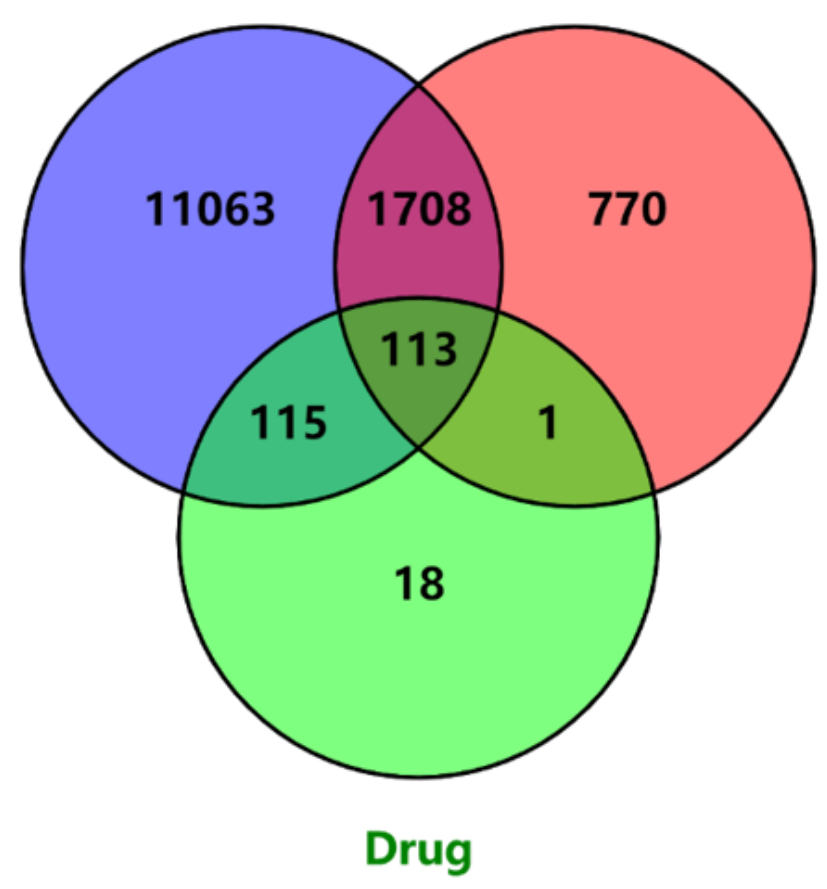

Figure 3

Venn diagram of common targets among CHSGP, insomnia and depression

A

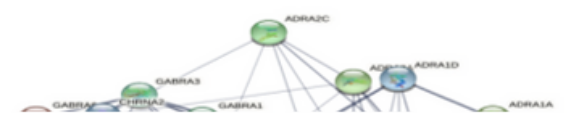

B

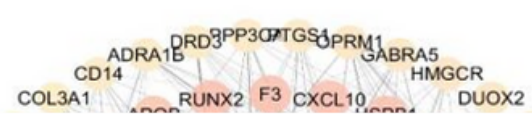

\section{Figure 4}

PPI network diagram of common targets among CHSGP, insomnia and depression. (A) PPI network diagram exported from the STRING website. (B) PPI network diagram drawn by Cytoscape software. 


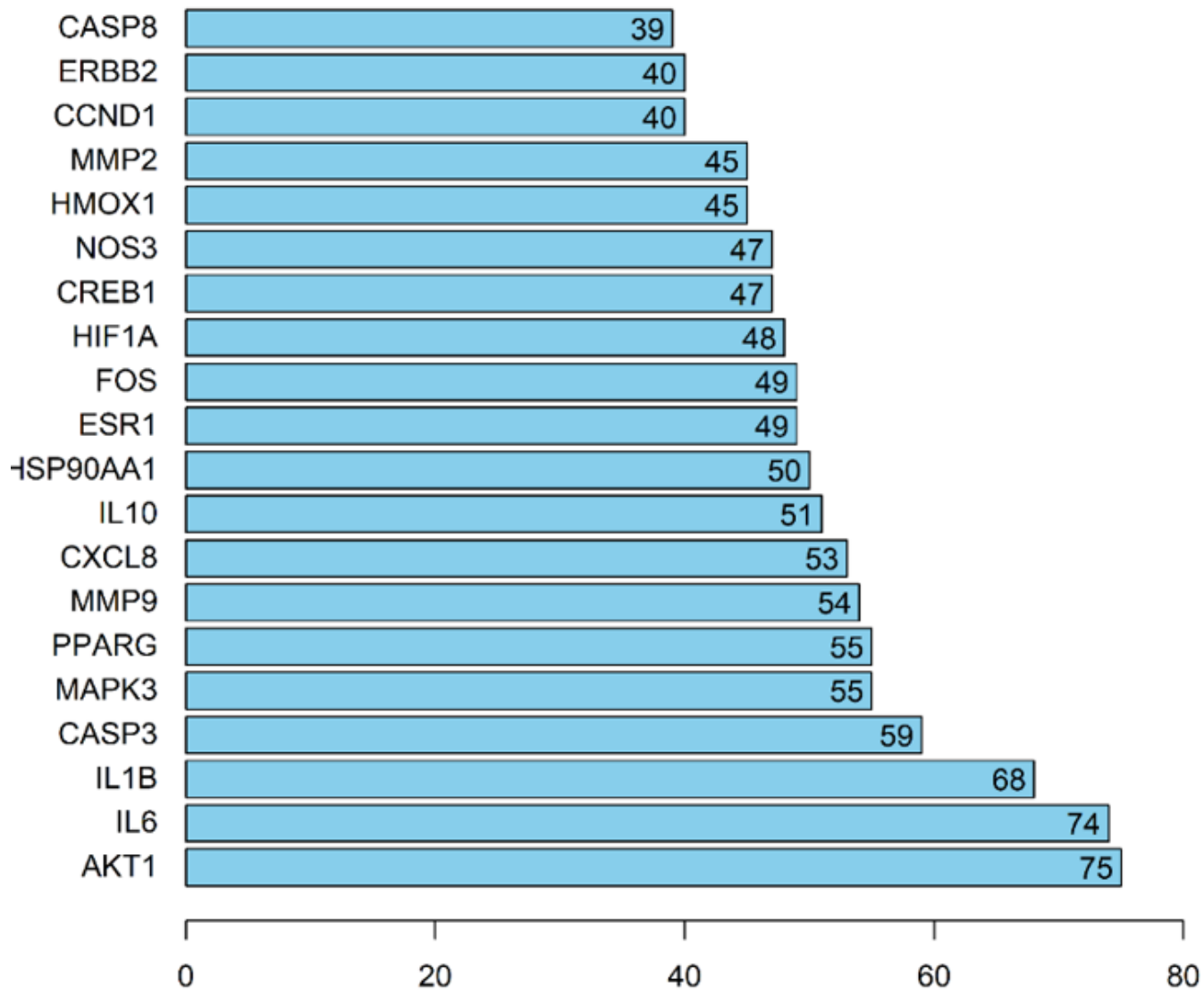

Figure 5

Topological analysis of common targets among CHSGP, insomnia and depression 


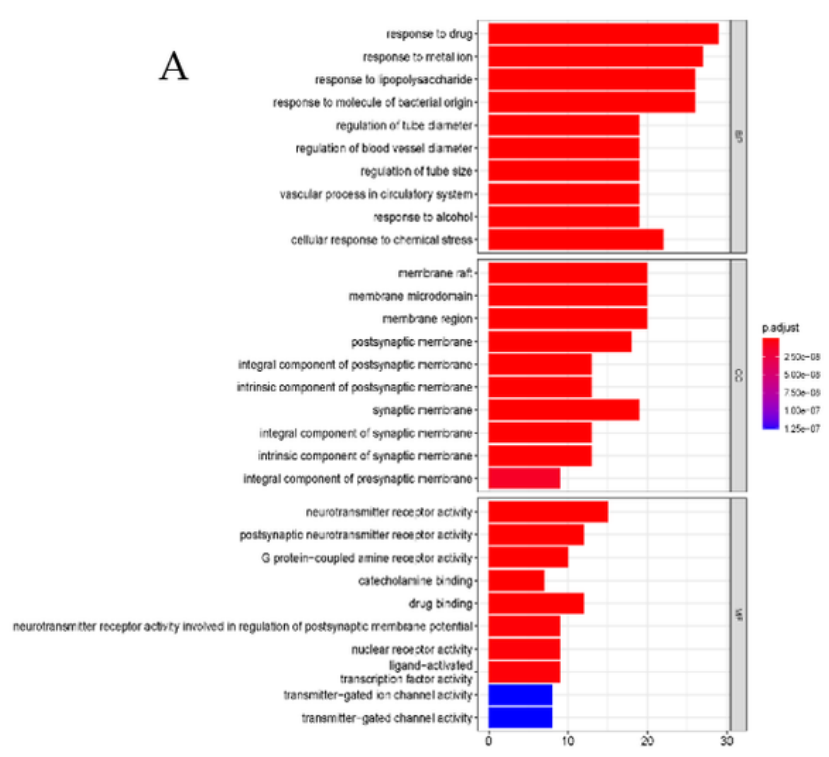

B
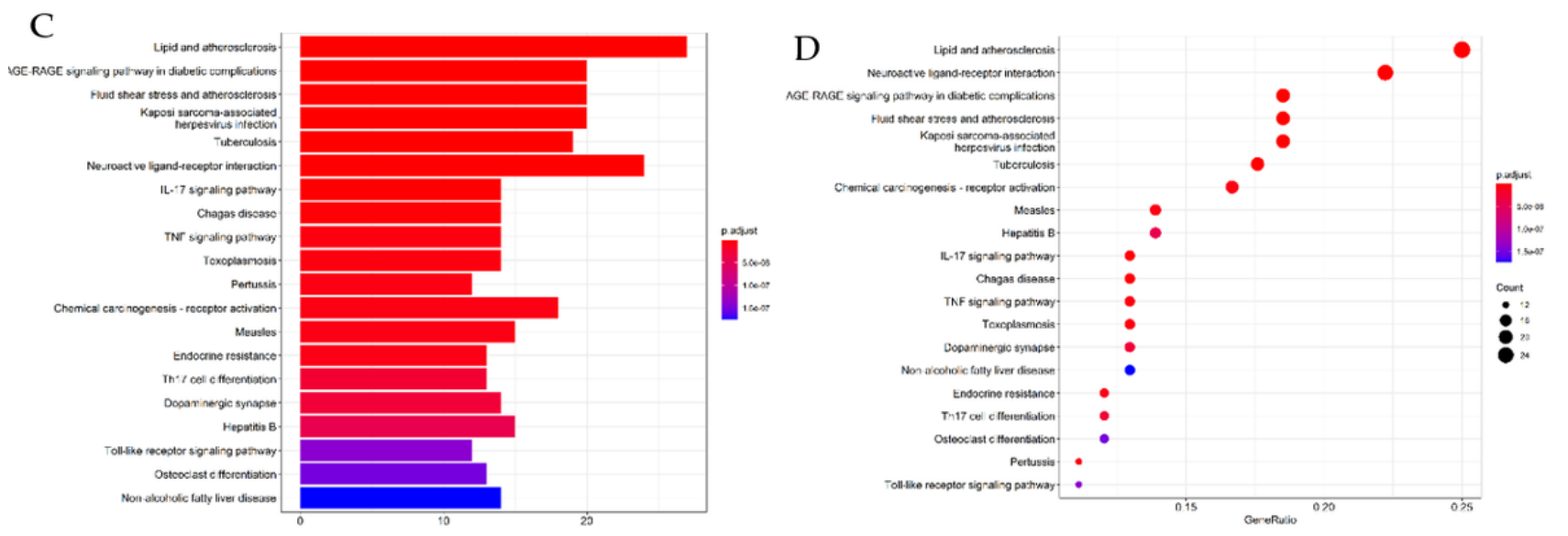

Figure 6

The histogram and bubble chart of GO enrichment and KEGG pathway analysis among CHSGP and insomnia and depression. (A)The histogram of GO enrichment analysis(BP, MF, CC). (B) The bubble of GO enrichment analysis (BP, MF, CC). (C)The histogram of KEGG pathway analysis. (D)The bubble of KEGG pathway analysis.

2

Figure 7

CHSGP and insomnia, depression component-disease-pathway-target network construction 


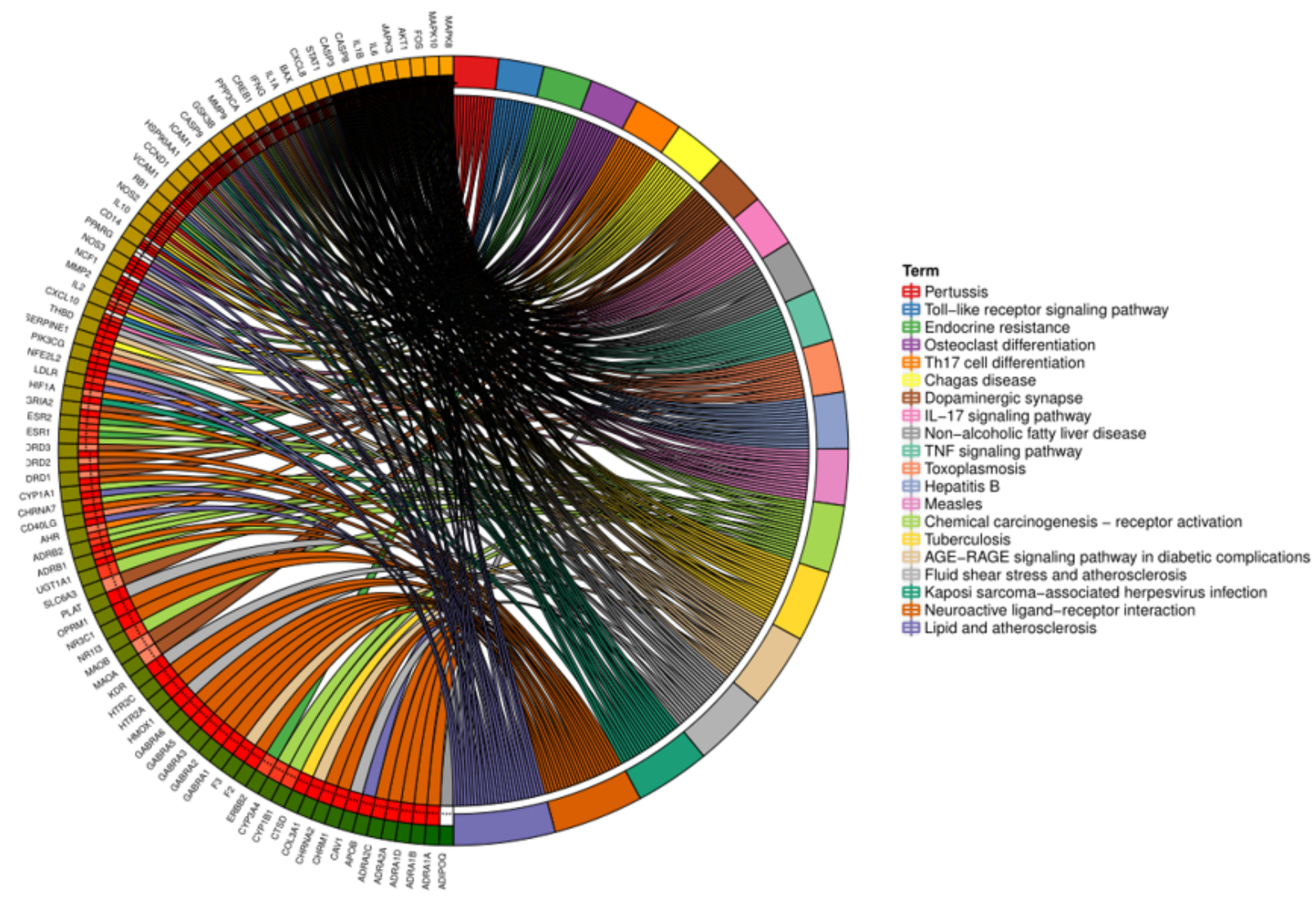

Figure 8

Chord diagram of the relationship in the KEGG pathways and targets among CHSGP, insomnia and depression 


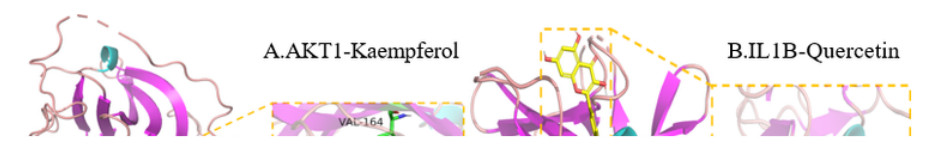

\section{Figure 9}

3D molecular docking diagrams of active ingredients and targets. (A) AKT1 and Kaempferol, (B) IL1B and quercetin, (C) IL6 and kaempferol, (D) AKT1 and stigmasterol, (E) GSK3B and luteolin, (F) GSK3B and 7-methoxy-2-methyl-isoflavone, and (G) GSK3B and beta-sitosterol. 